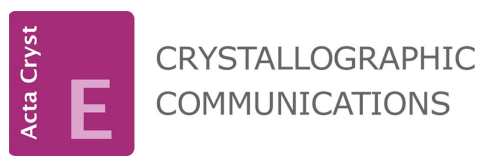

ISSN 2056-9890

Received 21 October 2015

Accepted 5 January 2016

Edited by A. J. Lough, University of Toronto, Canada

‡ Deceased July 7, 2015.

Keywords: crystal structure; polytypes; polymorphs; Sandmeyer; isocyanide; $\mathrm{N} \cdots \mathrm{Br}$ contacts; C...Br contacts

CCDC references: $1445499 ; 1445498$; 1445497; 1445496

Supporting information: this article has supporting information at journals.iucr.org/e
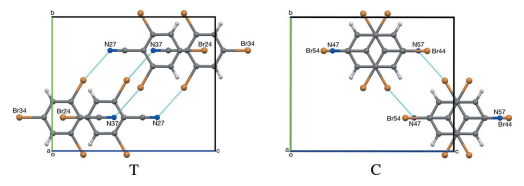

OPEN $\odot$ ACCESS

\section{Two new polytypes of 2,4,6-tribromobenzonitrile}

\author{
Doyle Britton, $¥$ Wayland E. Noland* and Kenneth J. Tritch
}

Department of Chemistry, University of Minnesota, Minneapolis, MN 55455-0431, USA. *Correspondence e-mail: nolan001@umn.edu

Three polymorphs of 2,4,6-tribromobenzonitrile ( $\mathrm{RCN}), \mathrm{C}_{7} \mathrm{H}_{2} \mathrm{Br}_{3} \mathrm{~N}$, two of which are novel and one of which is a redetermination of the original structure first determined by Carter \& Britton [(1972). Acta Cryst. B28, 945-950] are found to be polytypic. Each has a layer structure which differs only in the stacking of the layers. Each layer is composed of molecules associated through $\mathrm{C} \equiv \mathrm{N} \cdots \mathrm{Br}$ contacts which form $R_{2}^{2}(10)$ rings. Two such rings are associated with each $\mathrm{N}$ atom; one with each ortho-Br atom. No new polytypes of 1,3,5-tribromo2-isocyanobenzene (RNC) were found but a re-determination of the original structure by Carter et al. [(1977). Cryst. Struct. Commun. 6, 543-548] is presented. RNC was found to be isostructural with one of the novel polytypes of RCN. Unit cells were determined for $23 \mathrm{RCN}$ samples and $11 \mathrm{RNC}$ samples. Polytypes could not be distinguished based on crystal habits. In all four structures, each molecule of the asymmetric unit lies across a mirror plane.

\section{Chemical context}

The reported structures of 2,4,6-tribromobenzonitrile ( $\mathrm{RCN}$, Figs. 1 and 2; Carter \& Britton, 1972) and 1,3,5-tribromo-2isocyanobenzene (RNC, Figs. 1 and 3; Carter et al., 1977) have two-dimensional layers of similarly arranged molecules, but the packing of adjacent layers is distinctly different. At the time, no explanation was offered. It was puzzling, given that the two compounds are isoelectronic, isosteric, and the principal intermolecular interactions, $\mathrm{C} \equiv \mathrm{N} \cdots \mathrm{Br}$ and $\mathrm{N} \equiv \mathrm{C} \cdots \mathrm{Br}$, are similar. Recent reports of polytype organic structures, such as picryl bromide (Parrish et al., 2008) and 5,6-dimethylbenzofurazan 1-oxide (Britton et al., 2012) led to the idea that RCN and RNC might occur as polytypes. Earlier, Bredig (1930) had determined the space group and unit cell of RCN with the same results as Carter \& Britton. Bredig was trying to follow up on the goniometer studies of Jaeger (1909), but while he found the same $a: b$ ratio as Jaeger in the RCN unit cell, he found a different $b: c$ ratio.<smiles>N#Cc1c(Br)cc(Br)cc1Br</smiles><smiles>N#Cc1c(Br)cc(Br)cc1Br</smiles>

Accordingly, a search was made for polytypes of RCN, and to a lesser extent, of RNC. Four different structures were identified. RCN-I is the original $Z=2$ structure of RCN; RCNII is a new $Z=8$ polytype; RCN-III is a new $Z=12$ polytype. No RNC counterparts to RCN-I or RCN-III were observed. 
<smiles>Nc1c(Br)cc(Br)cc1Br</smiles><smiles>C[C@H](O)[C@H](O)O[N+](=O)[O-]</smiles><smiles>CC(=O)OCC(C)OC(C)C</smiles><smiles>O=CNc1c(Br)cc(Br)cc1Br</smiles>

1. DIPEA, DCE 2. $\mathrm{POCl}_{3}$

Figure 1

Synthesis of RCN and RNC.

RNC-II is the original $Z=8$ structure. As the $Z$ values suggest, $\mathrm{RCN}-\mathrm{II}$ and RNC-II are isomorphs.

\section{Structural commentary}

Molecules of RCN and RNC are nearly planar. The average distance of atoms from the plane of best fit is $0.025 \AA$ in RCNI. For RCN-II, the average distances are 0.037 and $0.010 \AA$, for the (N27) and (N37) molecules, respectively. In RNC-II, the molecules are slightly more distorted, with average deviations of 0.043 and $0.017 \AA$ for the (N127) and (N137) molecules, respectively. For RCN-III, the average distances are 0.009 , 0.018 , and $0.032 \AA$ for the (N47), (N57), and (N67) molecules, respectively.

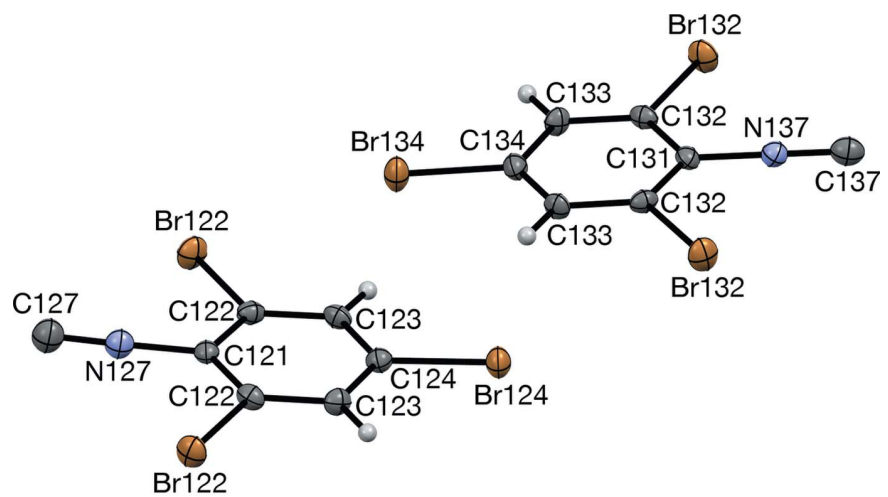

RNC-II

Figure 3

Molecular structure, with atom labeling, of RNC-II viewed along [120]. Displacement ellipsoids are drawn at the $50 \%$ probability level. Each molecule lies across a crystallographic mirror plane.

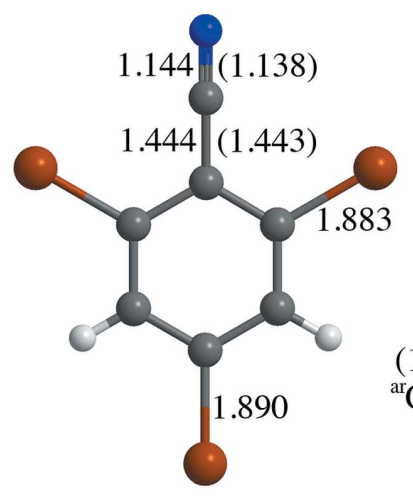

$\mathrm{RCN}$

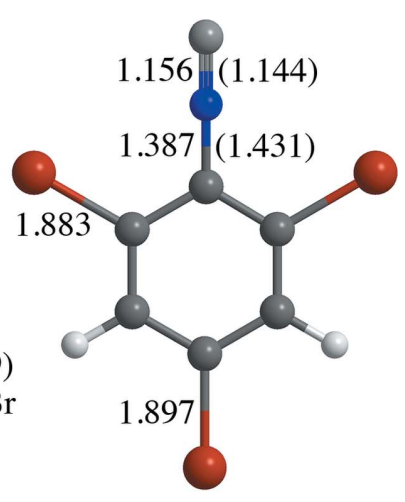

RNC
Figure 4

Selected bond lengths ( $⿱$ ) in RCN and RNC, averaged across all polytypes. The data shown in parentheses are the mean distances for each bond type reported by Allen et al. (1987).

The bond lengths in RCN and $\mathrm{RNC}$ are generally similar (Fig. 4). They are also similar to the mean bond distances reported for bonds of each type (Allen et al., 1987). The $\mathrm{N}$

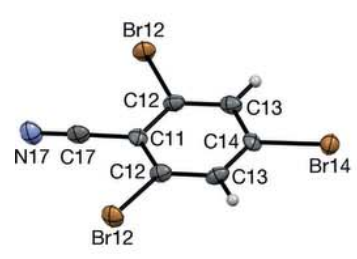

$\mathrm{RCN}-\mathrm{I}$

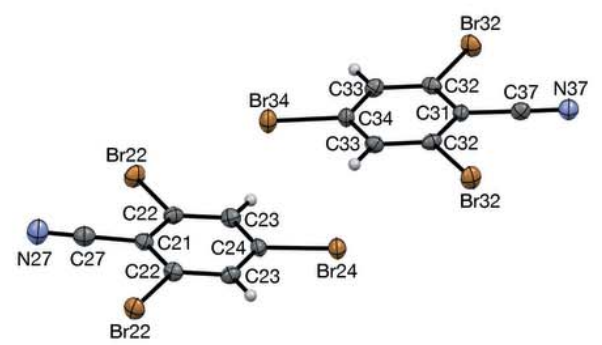

$\mathrm{RCN}-\mathrm{II}$

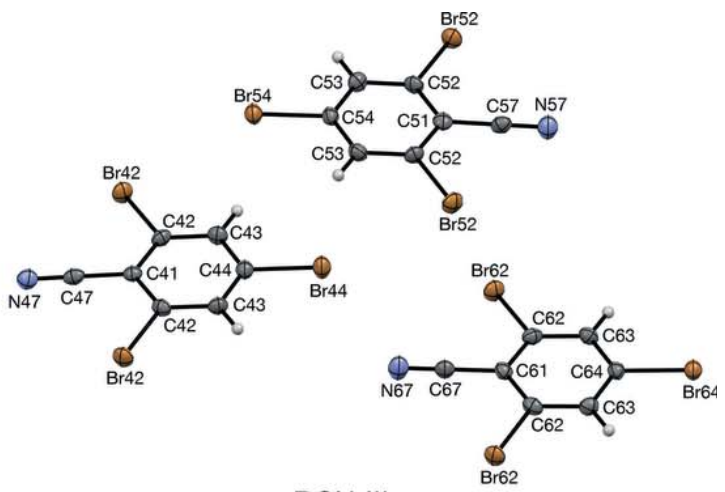

RCN-III

Figure 2

Molecular structures, with atom labeling, of RCN-I viewed along [111]]; RCN-II viewed along [120]; RCN-III viewed along [120]. Displacement ellipsoids are drawn at the $50 \%$ probability level. In discussion, molecules are named by their respective nitrogen atoms. Each molecule lies across a crystallographic mirror plane. 


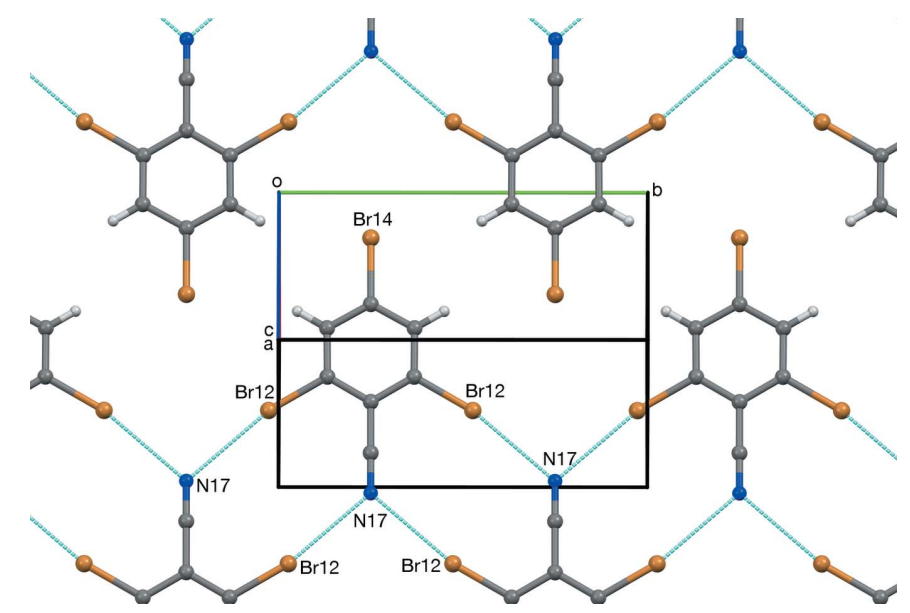

Figure 5

View of one layer of RCN-I along [101] . Dashed blue lines represent short contacts.

atom in RNC is displaced toward the aryl ring compared to the literature distances for aryl isocyanides.

\section{Supramolecular features}

Fig. 5 shows a two-dimensional layer of RCN-I. All of the structures are composed of similar layers. Adjacent molecules are associated through $\mathrm{C} \equiv \mathrm{N} \cdots \mathrm{Br}$ interactions, arranged in $R_{2}^{2}(10)$ rings (Etter, 1990; Bernstein et al., 1995). The $\mathrm{CN} \cdots \mathrm{Br}$ distances in these rings range between 3.053 and $3.077 \AA$ (Table 1); these distances can be compared with the $\mathrm{N} \cdots \mathrm{Br}$ van der Waals distance of $3.40 \AA$ (Bondi, 1964; Rowland \& Taylor, 1996). Each layer in RCN-II is composed of alternating (N27) and (N37) molecules. RCN-III contains two layers of alternating (N47) and (N57) molecules for each layer composed entirely of (N67) molecules. Adjacent pairs of layers show translational or pseudotranslational, or pseudocentric stacking (Fig. 6). RCN-I shows translational stacking between all adjacent layers (Fig. 7). In RCN-II, alternating pairs of layers show pseudocentric and pseudotranslational stacking (Fig. 8). In RCN-III, each layer of (N67) molecules pseudotranslationally overlaps both neighboring (N47/N57) layers, while pairs of adjacent (N47/N57) layers, every third pair of layers, overlap pseudocentrically (Fig. 9).

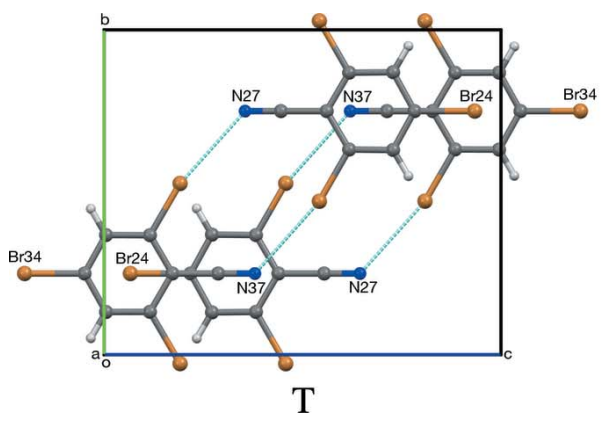

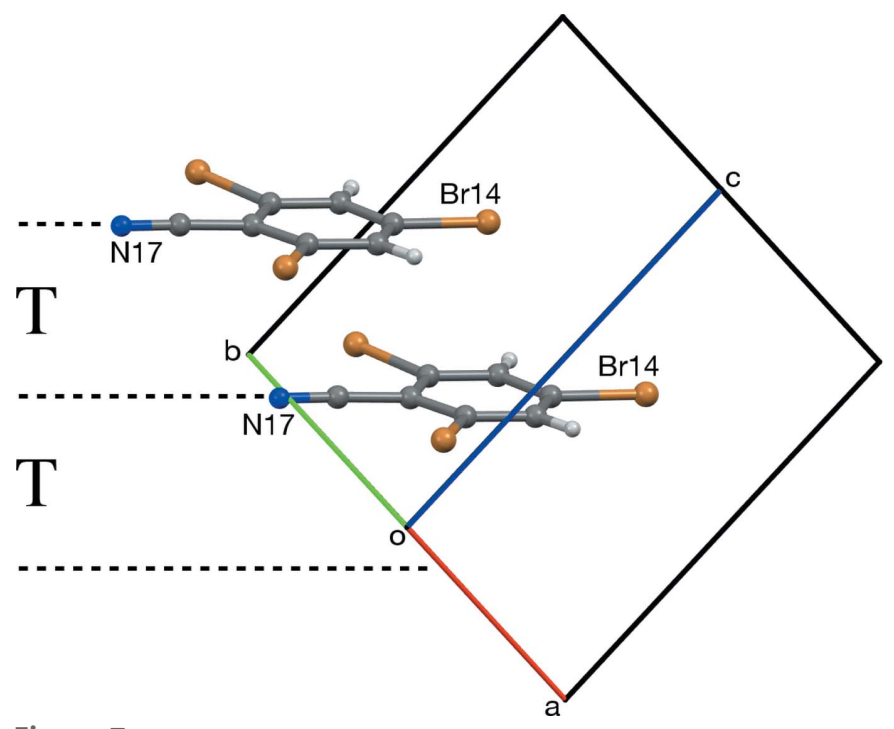

Figure 7

Translational ( $\mathrm{T}$ ) stacking of layers in $Z=2 \mathrm{RCN}-\mathrm{I}$, viewed along [110]. If the unit cell of RCN-I is transformed by the matrix [100/010/201], the dimensions of the projection become 10.247 (3) $\times 12.480$ (3) $\AA$, which is similar to the corresponding $b \times c$ measurements, $10.2147(10) \times$ 12.4754 (12) Å for RCN-II, and 10.2167 (18) × 12.493 (2) A for RCN-III.

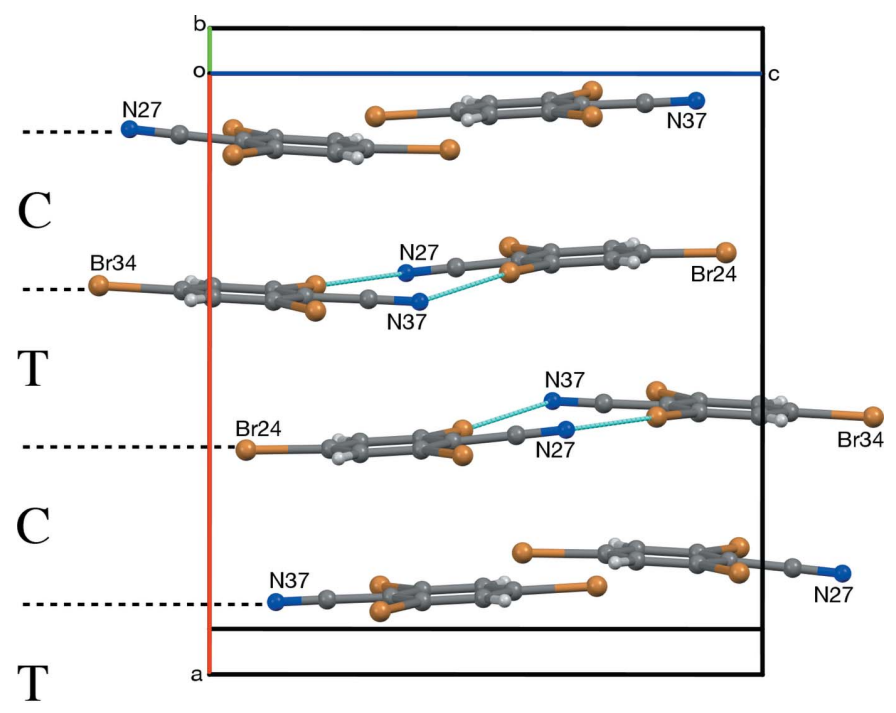

Figure 8

Pseudocentric (C) and pseudotranslational (T) stacking of layers in $Z=8$ RCN-II, viewed roughly along [010].

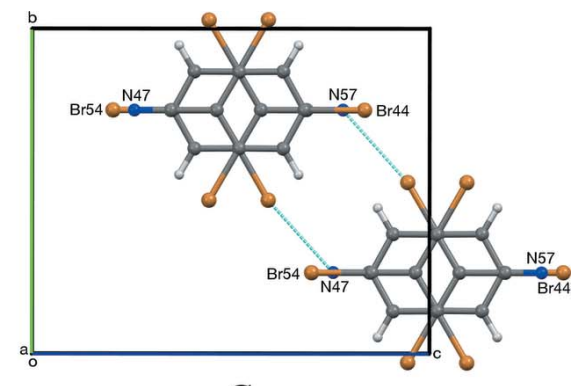

C

Figure 6

Pseudotranslational (T) and pseudocentric (C) stacking of layers in RCN-II and RCN-III, respectively. Both are viewed along [100]. The molecules shown are the second pair of layers from the top, in Fig. 7 and Fig. 8, respectively. 


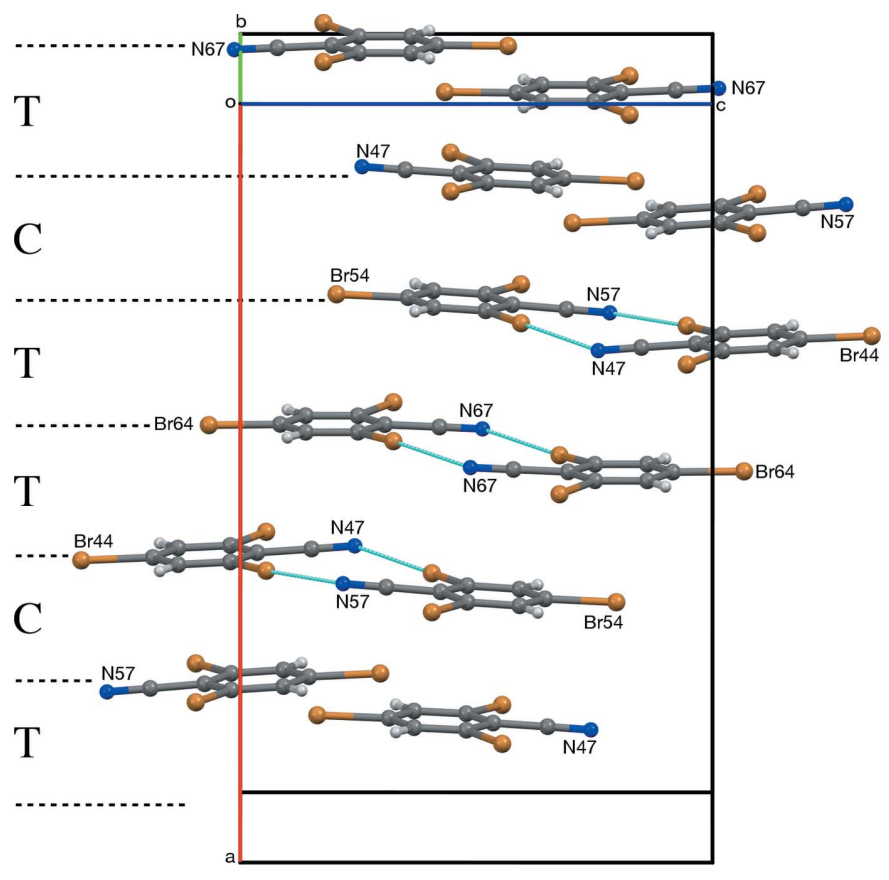

Figure 9

Pseudotranslational (T) and pseudocentric (C) stacking of layers in $Z=$ 12 RCN-III, viewed roughly along [010].

The NC..Br contact distances in RNC-II are a smaller percentage of the van der Waals distance, $3.63 \AA$, versus corresponding atoms in RCN-II. The contacts in RNC-II occur at slightly wider angles than those in RCN-II (Table 1).

In RCN-II, the planes of best fit of the two different molecules are inclined by $6.5^{\circ}$ to each other; in RNC-II this inclination is $7.5^{\circ}$. In $\mathrm{RCN}-\mathrm{III}$, the relative inclination of planes of (N47) and (N57) molecules is $7.0^{\circ}$. These two planes are approximately bisected by the planes of (N67) molecules.

\section{Database survey}

A search of the Cambridge Structural Database (Version 5.36, update 3; Groom \& Allen, 2014) for 2,4,6-trihalo-3,5-unsubstituted benzonitriles found nine entries: RCN; its trichloro analog, Gol'der et al. (1952), Carter \& Britton (1972), Pink et al. (2000); its trifluoro analog, Britton (2008); four mixedhalogen entries, Gleason \& Britton (1978), Britton (2005), Britton et al. (2002), and Britton (1997). Searching for the corresponding isocyanides found two entries: RNC and its trichloro analog (Pink et al., 2000).

Layers of the type observed in $\mathrm{RCN}$ were reported in 2,6dibromo entries with $\mathrm{Cl}, \mathrm{Br}$, or $\mathrm{I}$ at the 4-position. Other entries exhibit short contacts between the cyano- or isocyanogroup and one ortho-halogen atom of an intralayer molecule, with various interlayer contacts. Polymorphs are only reported for 2,4,6-trichlorobenzonitrile; those are not polytypic.

Expanding the search to include organometallic complexes found three more entries, with the cyano $\mathrm{N}$ or isocyano $\mathrm{C}$ atom ligating gallium (trifluorobenzonitrile; Tang et al., 2012),
Table 1

Short contact geometry $\left(\AA,^{\circ}\right)$.

\begin{tabular}{llll}
\hline$X \equiv Y \cdots \mathrm{Br}$ & $X \equiv Y$ & $Y \cdots \mathrm{Br}$ & $X \equiv Y \cdots \mathrm{Br}$ \\
\hline $\mathrm{C} 17 \equiv \mathrm{N} 17 \cdots \mathrm{Br} 12^{\mathrm{i}}$ & $1.144(10)$ & $3.053(4)$ & $131.45(9)$ \\
$\mathrm{C} 27 \equiv \mathrm{N} 27 \cdots \mathrm{Br} 32^{\mathrm{ii}}$ & $1.132(7)$ & $3.059(3)$ & $131.76(7)$ \\
$\mathrm{N} 127 \equiv \mathrm{C} 127 \cdots \mathrm{Br} 132^{\mathrm{ii}}$ & $1.147(6)$ & $3.141(4)$ & $134.01(8)$ \\
$\mathrm{C} 37 \equiv \mathrm{N} 37 \cdots \mathrm{Br} 22^{\mathrm{iii}}$ & $1.156(6)$ & $3.077(3)$ & $130.68(10)$ \\
$\mathrm{N} 137 \equiv \mathrm{C} 137 \cdots \mathrm{Br} 122^{\mathrm{iii}}$ & $1.164(6)$ & $3.161(4)$ & $133.23(11)$ \\
$\mathrm{C} 47 \equiv \mathrm{N} 47 \cdots \mathrm{Br} 52^{\mathrm{ii}}$ & $1.146(6)$ & $3.072(3)$ & $130.95(9)$ \\
$\mathrm{C} 57 \equiv \mathrm{N} 57 \cdots \mathrm{Br} 42^{\mathrm{iii}}$ & $1.147(6)$ & $3.057(3)$ & $131.47(7)$ \\
$\mathrm{C} 67 \equiv \mathrm{N} 67 \cdots \mathrm{Br} 62^{\mathrm{iv}}$ & $1.139(6)$ & $3.065(3)$ & $131.96(7)$ \\
\hline
\end{tabular}

Symmetry codes: (i) $-x, 1-y,-z$; (ii) $x, y,-1+z$; (iii) $x, y, 1+z$; (iv) $1-x, 1-y$, $1-z$.

rhenium (trichloroisocyanobenzene; Ko et al., 2011), and ruthenium (RNC; Leung et al., 2009).

\section{Synthesis and crystallization}

2,4,6-Tribromoaniline was prepared from aniline according to the work of Coleman \& Talbot (1943).

RCN, adapted from the work of Toya et al. (1992): Diazotization: 2,4,6-Tribromoaniline (1.25 g), water $(2.5 \mathrm{ml})$, and glacial acetic acid $(4.4 \mathrm{ml})$ were combined in a roundbottomed flask. The resulting suspension was cooled in an ice bath, and then $\mathrm{H}_{2} \mathrm{SO}_{4}(98 \%, 1.0 \mathrm{ml})$ was added dropwise, followed by an ice-cold solution of $\mathrm{NaNO}_{2}(520 \mathrm{mg})$ in water $(4 \mathrm{ml})$. The resulting mixture was warmed to $310 \mathrm{~K}$ for $1 \mathrm{~h}$, and then cooled in an ice bath. Cyanide suspension: $\mathrm{CuCN}$ (680 mg) and $\mathrm{NaCN}$ (1.12 g) were dissolved in water $(20 \mathrm{ml})$. $\mathrm{NaHCO}_{3}(10.9 \mathrm{~g})$ and ethyl acetate $(10 \mathrm{ml})$ were added, giving a suspension, which was cooled in an ice bath. Cyanation: The diazotization mixture was added dropwise to the cyanide suspension as quickly as possible without causing excessive foaming. The ice bath was removed and then the mixture was stirred overnight. The organic phase was set aside. The aqueous phase was extracted with ethyl acetate $(3 \times 10 \mathrm{ml})$. The combined organic portions were washed with brine $(10 \mathrm{ml})$, dried with $\mathrm{Na}_{2} \mathrm{SO}_{4}$, and concentrated at reduced pressure, giving a brown powder, which was purified by column chromatography $\left(\mathrm{SiO}_{2}\right.$, hexane-ethyl acetate, gradient from 1:0 to $10: 1)$. The desired fraction $\left(R_{f}=0.61\right.$ in 8:1) was concentrated at reduced pressure, giving beige needles (760 mg, 59\%). M.p. 400-400.5 K (lit. $402 \mathrm{~K}$; Giumanini et al., 1996); ${ }^{1} \mathrm{H}$ NMR (300 MHz, $\left.\mathrm{CD}_{2} \mathrm{Cl}_{2}\right) \delta 7.853(s, \mathrm{H} 13) ;{ }^{13} \mathrm{C} \mathrm{NMR}$ (75 MHz, $\mathrm{CD}_{2} \mathrm{Cl}_{2}$ ) $\delta 135.3$ (C13), 128.6 (C14), 127.4 (C12), 118.3 (C17), 116.0 (C11); IR ( NaCl, cm $\left.{ }^{-1}\right)$ 3095, 3068, 2921 (w), 2233 ( $s, \mathrm{C} \equiv \mathrm{N}$; lit. 2232), $1716(w), 1563(s), 1527$ (s), 1431 $(s), 1410(s), 1370(s), 1353(s), 1328,1191(s), 1109(s), 1087$, $1063(s), 854(s), 809(s), 748(s)$; MS (EI, $m / z)[M]^{+}$calculated for $\mathrm{C}_{7} \mathrm{H}_{2} \mathrm{Br}_{3} \mathrm{~N}$ 336.7732, found 336.7716.

2,4,6-Tribromoformanilide, adapted from the work of Krishnamurthy (1982): Acetic anhydride (3.2 ml) and tetrahydrofuran (THF, $5.0 \mathrm{ml}$ ) were combined in a roundbottomed flask. Formic acid (88\% aq., $1.7 \mathrm{ml})$ was added dropwise. The resulting solution was stirred for $30 \mathrm{~min}$ at room temperature. A solution of 2,4,6-tribromoaniline (1.82 $\mathrm{g}$ ) in 
Table 2

Experimental details.

\begin{tabular}{|c|c|c|c|c|}
\hline & $\mathrm{RCN}-\mathrm{I}$ & RCN-II & RCN-III & RNC-II \\
\hline \multicolumn{5}{|l|}{ Crystal data } \\
\hline Chemical formula & $\mathrm{C}_{7} \mathrm{H}_{2} \mathrm{Br}_{3} \mathrm{~N}$ & $\mathrm{C}_{7} \mathrm{H}_{2} \mathrm{Br}_{3} \mathrm{~N}$ & $\mathrm{C}_{7} \mathrm{H}_{2} \mathrm{Br}_{3} \mathrm{~N}$ & $\mathrm{C}_{7} \mathrm{H}_{2} \mathrm{Br}_{3} \mathrm{~N}$ \\
\hline$M_{\mathrm{r}}$ & 339.83 & 339.83 & 339.83 & 339.83 \\
\hline Temperature $(\mathrm{K})$ & 173 & 173 & 173 & 173 \\
\hline$a, b, c(\AA)$ & $\begin{array}{l}4.8742(15), 10.247(3) \\
\quad 8.683(3)\end{array}$ & $\begin{array}{l}13.6183(13), 10.2147(10), \\
\quad 12.4754(12)\end{array}$ & $\begin{array}{l}20.399(4), 10.2167(18) \\
\quad 12.493(2)\end{array}$ & $\begin{array}{l}13.5916(18), 10.1464(13), \\
12.6158(16)\end{array}$ \\
\hline$\alpha, \beta, \gamma\left(^{\circ}\right)$ & $90,94.97(1), 90$ & $90,90,90$ & $90,90,90$ & $90,90,90$ \\
\hline Radiation type & Mo $K \alpha$ & Mo $K \alpha$ & Mo $K \alpha$ & Mo $K \alpha$ \\
\hline$\mu\left(\mathrm{mm}^{-1}\right)$ & 13.93 & 13.88 & 13.87 & 13.84 \\
\hline Crystal size $(\mathrm{mm})$ & $0.50 \times 0.15 \times 0.10$ & $0.25 \times 0.20 \times 0.07$ & $0.50 \times 0.15 \times 0.10$ & $0.40 \times 0.35 \times 0.20$ \\
\hline \multicolumn{5}{|l|}{ Data collection } \\
\hline Diffractometer & Bruker $1 \mathrm{~K}$ area detector & Bruker $1 \mathrm{~K}$ area detector & Bruker $1 \mathrm{~K}$ area detector & Bruker APEXII CCD \\
\hline$R_{\text {int }}$ & 0.127 & 0.052 & 0.055 & 0.078 \\
\hline$(\sin \theta / \lambda)_{\max }\left(\AA^{-1}\right)$ & 0.649 & 0.650 & 0.616 & 0.650 \\
\hline \multicolumn{5}{|l|}{ Refinement } \\
\hline$R\left[F^{2}>2 \sigma\left(F^{2}\right)\right], w R\left(F^{2}\right), S$ & $0.046,0.116,1.01$ & $0.028,0.063,1.02$ & $0.023,0.046,1.07$ & $0.025,0.055,1.06$ \\
\hline No. of reflections & 1024 & 2093 & 2691 & 2105 \\
\hline No. of parameters & 58 & 115 & 173 & 116 \\
\hline H-atom treatment & $\begin{array}{l}\text { H-atom parameters } \\
\text { constrained }\end{array}$ & $\begin{array}{l}\text { H-atom parameters } \\
\text { constrained }\end{array}$ & $\begin{array}{l}\text { H-atom parameters } \\
\text { constrained }\end{array}$ & $\begin{array}{l}\text { H-atom parameters } \\
\text { constrained }\end{array}$ \\
\hline$\Delta \rho_{\max }, \Delta \rho_{\min }\left(\mathrm{e} \AA^{-3}\right)$ & $1.36,-1.28$ & $0.44,-0.69$ & $0.56,-0.49$ & $0.44,-0.48$ \\
\hline
\end{tabular}

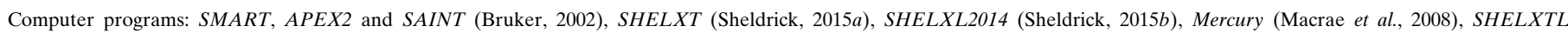
(Sheldrick, 2008), enCIFer (Allen et al., 2004), and publCIF (Westrip, 2010).

THF (20 ml) was added dropwise. The resulting mixture was stirred for $18 \mathrm{~h}$. The resulting heterogeneous mixture was filtered through neutral alumina (Sigma-Aldrich 199974, $5 \mathrm{~cm} \mathrm{H} \times 3 \mathrm{~cm} \mathrm{D)}$, with addition of sufficient THF to elute all product, as indicated by TLC. The filtrate was concentrated at reduced pressure. The resulting residue was washed with sat. $\mathrm{NaHCO}_{3}$ solution $(50 \mathrm{ml})$, and then filtered. The filter cake was recrystallized from acetone, giving white needles $(1.72 \mathrm{~g}$, 87\%). M.p. 493-494 K (lit. 494.5 K; Chattaway et al., 1899); $R_{f}$ $=0.48\left(\mathrm{SiO}_{2}\right.$ in 1:1 hexane-ethyl acetate $) ;{ }^{1} \mathrm{H}$ NMR $(300 \mathrm{MHz}$, $\left.\left(\mathrm{CD}_{3}\right)_{2} \mathrm{SO}\right) \delta 10.192(s, \mathrm{~N} H, O-E$ conformer, $0.87 \mathrm{H}), 8.522(s$, $\mathrm{NH}, O-Z$ conformer, $0.13 \mathrm{H}), 8.260(s, \mathrm{CHO}, 1 \mathrm{H}), 8.018(\mathrm{~s}, \mathrm{CH}$, $2 \mathrm{H}) ;{ }^{13} \mathrm{C}$ NMR $\left(75 \mathrm{MHz},\left(\mathrm{CD}_{3}\right)_{2} \mathrm{SO}\right) \delta 165.9$ (CO, O-Z conformer), 159.8 (CO, $O$-E conformer), 134.6 (ipso- $C$ ), 134.4 $(C \mathrm{H}), 124.5$ (ortho-CBr), 121.1 (para-CBr); IR $\left(\mathrm{NaCl}, \mathrm{cm}^{-1}\right.$ ) 3201, 3166, 1661 ( $s, \mathrm{C}=\mathrm{O}), 1558,1154,858,810$; MS (ESI, $m / z)[M-\mathrm{H}]^{-}$calculated for $\mathrm{C}_{7} \mathrm{H}_{4} \mathrm{Br}_{3} \mathrm{NO} 355.7750$, found 355.7758. Analysis (MHW Laboratories, Phoenix, AZ, USA) calculated for $\mathrm{C}_{7} \mathrm{H}_{4} \mathrm{Br}_{3} \mathrm{NO}$ : C 23.50, $\mathrm{H}$ 1.13, $\mathrm{Br} 66.99$, N 3.91; found $\mathrm{C} 23.42, \mathrm{H} 1.15, \mathrm{Br} 66.71, \mathrm{~N} 3.57$.

RNC, adapted from the work of Ugi et al. (1965): 2,4,6Tribromoformanilide (1.96 g) and $N, N$-diisopropylethylamine (DIPEA, $3.4 \mathrm{ml})$ were added to 1,2-dichloroethane $(75 \mathrm{ml})$. The resulting suspension was refluxed for $5 \mathrm{~min}$, and then cooled to room temperature. $\mathrm{POCl}_{3}(0.6 \mathrm{ml})$ was added dropwise. The mixture was stirred for $18 \mathrm{~h}$, cooled in an ice bath, and then filtered through neutral alumina $(3 \mathrm{~cm} \mathrm{H} \times$ $3 \mathrm{~cm} \mathrm{D}$ ), with addition of sufficient dichloromethane (DCM) to elute all product as indicated by TLC. The filtrate was concentrated at reduced pressure. The resulting yellow residue was dissolved in DCM $(25 \mathrm{ml})$, cooled in an ice bath, and washed with ice-cold acetic acid solution $(0.025 \mathrm{M}, 3 \times 15 \mathrm{ml})$, and then ice-cold sat. $\mathrm{NaHCO}_{3}$ solution $(15 \mathrm{ml})$. The organic phase was collected, dried with $\mathrm{Na}_{2} \mathrm{SO}_{4}$, and then concentrated under a stream of nitrogen, giving beige needles upon filtration (630 mg, 34\%). M.p. $390 \mathrm{~K}$ (lit. $394 \mathrm{~K}$, Mironov \& Mokrushin, 1999); $R_{f}=0.75\left(\mathrm{Al}_{2} \mathrm{O}_{3}\right.$ in 2:1 hexane-ethyl acetate); ${ }^{1} \mathrm{H}$ NMR (300 MHz, $\left.\mathrm{CD}_{2} \mathrm{Cl}_{2}\right) \delta 7.827$ (s, H123); ${ }^{13} \mathrm{C}$ NMR (75 MHz, $\left.\left(\mathrm{CD}_{3}\right)_{2} \mathrm{CO}\right) 159.7$ (C127), 135.8 (C123), 135.4 (C121), 124.5 (C124), 122.0 (C122); IR (NaCl, cm ${ }^{-1}$ ) 3162, 3068, 2921, 2128 ( $s, \mathrm{~N} \equiv \mathrm{C}$; lit. 2125), $1660(s), 1555(s), 1370$ $(s), 856(s), 701(s)$; MS (EI, $m / z)[M]^{+}$calculated for $\mathrm{C}_{7} \mathrm{H}_{2} \mathrm{Br}_{3} \mathrm{~N}$ 336.7732, found 336.7734 .

Crystallization: RCN crystals were grown by slow evaporation of single-solvent solutions (290-295 K). RCN-I was obtained from acetonitrile, benzene, chloroform, or methylene chloride; RCN-II from mesitylene; RCN-III from benzene or chloroform. RNC-II crystals were obtained by sublimation ( $385 \mathrm{~K}, 0.05$ torr), or by slow evaporation from the same solvents as RCN (268-295 K). 


\section{Refinement}

Crystal data, data collection, and structure refinement details for RCN and RNC are summarized in Table 2. H atoms were placed in calculated positions and refined as riding atoms, with $\mathrm{C}-\mathrm{H}=0.95 \AA$ and $U_{\text {iso }}(\mathrm{H})=1.2 U_{\text {eq }}(\mathrm{C})$.

\section{Acknowledgements}

The authors thank Victor G. Young, Jr. (X-Ray Crystallographic Laboratory, University of Minnesota) for assistance with unit cell and crystal determinations, and the Wayland E. Noland Research Fellowship Fund at the University of Minnesota Foundation for generous financial support of this project.

\section{References}

Allen, F. H., Johnson, O., Shields, G. P., Smith, B. R. \& Towler, M. (2004). J. Appl. Cryst. 37, 335-338.

Allen, F. H., Kennard, O., Watson, D. G., Brammer, L., Orpen, A. G. \& Taylor, R. (1987). J. Chem. Soc. Perkin Trans. 2, S1-19.

Bernstein, J., Davis, R. E., Shimoni, L. \& Chang, N.-L. (1995). Angew. Chem. Int. Ed. Engl. 34, 1555-1573.

Bondi, A. (1964). J. Phys. Chem. 68, 441-451.

Bredig, M. A. (1930). Z. Kristallogr. 74, 56-61.

Britton, D. (1997). Acta Cryst. C53, 225-227.

Britton, D. (2005). Acta Cryst. E61, o1726-o1727.

Britton, D. (2008). Acta Cryst. C64, o583-o585.

Britton, D., Noland, W. E. \& Henke, T. K. (2002). Acta Cryst. E58, o185-o187.

Britton, D., Young, V. G., Noland, W. E., Pinnow, M. J. \& Clark, C. M. (2012). Acta Cryst. B68, 536-542.

Bruker (2002). APEX2, SMART, SAINT, and SADABS. Bruker AXS, Inc., Madison, Wisconsin, USA.

Carter, V. B. \& Britton, D. (1972). Acta Cryst. B28, 945-950.

Carter, V. B., Britton, D. \& Gleason, W. G. (1977). Cryst. Struct. Commun. 6, 543-548.
Chattaway, F. D., Orton, K. J. P. \& Hurtley, W. H. (1899). Ber. Dtsch. Chem. Ges. 32, 3635-3638.

Coleman, G. H. \& Talbot, W. F. (1943). Org. Synth, Coll. Vol. 2, 592595.

Etter, M. C. (1990). Acc. Chem. Res. 23, 120-126.

Giumanini, A. G., Verardo, G., Geatti, P. \& Strazzolini, P. (1996). Tetrahedron, 52, 7137-7148.

Gleason, W. B. \& Britton, D. (1978). Cryst. Struct. Commun. 7, 365370.

Gol'der, G. A., Zhdanov, G. S. \& Umanskij, M. M. (1952). Russ. J. Phys. Chem. 26, 1434-1437.

Groom, C. R. \& Allen, F. H. (2014). Angew. Chem. Int. Ed. 53, 662671.

Jaeger, F. M. (1909). Z. Kristallogr. 46, 268-269.

Ko, C.-C., Siu, J. W.-K., Cheung, A. W.-Y. \& Yiu, S.-M. (2011). Organometallics, 30, 2701-2711.

Krishnamurthy, S. (1982). Tetrahedron Lett. 23, 3315-3318.

Leung, C.-F., Ng, S.-M., Xiang, J., Wong, W.-Y., Lam, M. H.-W., Ko, C.-C. \& Lau, T.-C. (2009). Organometallics, 28, 5709-5714.

Macrae, C. F., Bruno, I. J., Chisholm, J. A., Edgington, P. R., McCabe, P., Pidcock, E., Rodriguez-Monge, L., Taylor, R., van de Streek, J. \& Wood, P. A. (2008). J. Appl. Cryst. 41, 466-470.

Mironov, M. A. \& Mokrushin, V. S. (1999). Russ. J. Org. Chem. 35, 693-697.

Parrish, D. A., Deschamps, J. R., Gilardi, R. D. \& Butcher, R. J. (2008). Cryst. Growth Des. 8, 57-62.

Pink, M., Britton, D., Noland, W. E. \& Pinnow, M. J. (2000). Acta Cryst. C56, 1271-1273.

Rowland, R. S. \& Taylor, R. (1996). J. Phys. Chem. 100, 7384-7391.

Sheldrick, G. M. (2008). Acta Cryst. A64, 112-122.

Sheldrick, G. M. (2015a). Acta Cryst. A71, 3-8.

Sheldrick, G. M. (2015b). Acta Cryst. C71, 3-8.

Tang, S., Monot, J., El-Hellani, A., Michelet, B., Guillot, R., Bour, C. \& Gandon, V. (2012). Chem. Eur. J. 18, 10239-10243.

Toya, Y., Takagi, M., Nakata, H., Suzuki, N., Isobe, M. \& Goto, T. (1992). Bull. Chem. Soc. Jpn, 65, 392-395.

Ugi, I., Fetzer, U., Eholzer, U., Knupfer, H. \& Offermann, K. (1965). Angew. Chem. Int. Ed. Engl. 4, 472-484.

Westrip, S. P. (2010). J. Appl. Cryst. 43, 920-925. 


\section{supporting information}

Acta Cryst. (2016). E72, 178-183 [doi:10.1107/S2056989016000256]

Two new polytypes of 2,4,6-tribromobenzonitrile

Doyle Britton, Wayland E. Noland and Kenneth J. Tritch

Computing details

Data collection: SMART (Bruker, 2002) for RCN-I, RCN-II, RCN-III; APEX2 (Bruker, 2002) for RNC-II. For all compounds, cell refinement: SAINT (Bruker, 2002); data reduction: SAINT (Bruker, 2002). Program(s) used to solve structure: SHELXTL (Sheldrick, 2008) for RCN-I, RCN-II, RCN-III; SHELXT (Sheldrick, 2015a) for RNC-II. Program(s) used to refine structure: SHELXTL (Sheldrick, 2008) for RCN-I, RCN-II, RCN-III; SHELXL2014 (Sheldrick, 2015b) for RNC-II. For all compounds, molecular graphics: Mercury (Macrae et al., 2008); software used to prepare material for publication: SHELXTL (Sheldrick, 2008), enCIFer (Allen et al., 2004), and publCIF (Westrip, 2010).

(RCN-I) 2,4,6-Tribromobenzonitrile - polytype I

Crystal data

$\mathrm{C}_{7} \mathrm{H}_{2} \mathrm{Br}_{3} \mathrm{~N}$

$M_{r}=339.83$

Monoclinic, $P 2_{1} / m$

Hall symbol: -P $2 \mathrm{yb}$

$a=4.8742(15) \AA$

$b=10.247(3) \AA$

$c=8.683(3) \AA$

$\beta=94.97(1)^{\circ}$

$V=432.0(2) \AA^{3}$

$Z=2$

\section{Data collection}

Bruker $1 \mathrm{~K}$ area-detector diffractometer

Radiation source: fine-focus sealed tube Graphite monochromator $\omega$ scans

Absorption correction: multi-scan

(SADABS; Bruker, 2002)

$T_{\min }=0.080, T_{\max }=0.248$

\section{Refinement}

Refinement on $F^{2}$

Least-squares matrix: full

$R\left[F^{2}>2 \sigma\left(F^{2}\right)\right]=0.046$

$w R\left(F^{2}\right)=0.116$

$S=1.01$

1024 reflections

58 parameters

0 restraints
$F(000)=312$

$D_{\mathrm{x}}=2.612 \mathrm{Mg} \mathrm{m}^{-3}$

Mo $K \alpha$ radiation, $\lambda=0.71073 \AA$

Cell parameters from 2049 reflections

$\theta=2.4-27.4^{\circ}$

$\mu=13.93 \mathrm{~mm}^{-1}$

$T=173 \mathrm{~K}$

Needle, colorless

$0.50 \times 0.15 \times 0.10 \mathrm{~mm}$

4093 measured reflections

1024 independent reflections

856 reflections with $I>2 \sigma(I)$

$R_{\text {int }}=0.127$

$\theta_{\max }=27.5^{\circ}, \theta_{\min }=2.4^{\circ}$

$h=-6 \rightarrow 6$

$k=-13 \rightarrow 13$

$l=-11 \rightarrow 11$

Primary atom site location: structure-invariant direct methods

Secondary atom site location: difference Fourier map

Hydrogen site location: inferred from neighbouring sites

$\mathrm{H}$-atom parameters constrained 
$w=1 /\left[\sigma^{2}\left(F_{\mathrm{o}}^{2}\right)+(0.069 P)^{2}\right]$

where $P=\left(F_{\mathrm{o}}^{2}+2 F_{\mathrm{c}}{ }^{2}\right) / 3$

$(\Delta / \sigma)_{\max }=0.001$

$$
\Delta \rho_{\max }=1.36 \mathrm{e} \AA^{-3}
$$

$\Delta \rho_{\min }=-1.28 \mathrm{e} \AA^{-3}$

Fractional atomic coordinates and isotropic or equivalent isotropic displacement parameters $\left(\hat{A}^{2}\right)$

\begin{tabular}{lllll}
\hline & $x$ & $y$ & $z$ & $U_{\text {iso }} * U_{\text {eq }}$ \\
\hline Br12 & $0.33356(11)$ & $0.47324(5)$ & $0.18676(7)$ & $0.0280(2)$ \\
Br14 & $1.11323(14)$ & 0.7500 & $0.57820(9)$ & $0.0256(3)$ \\
N17 & $-0.0263(14)$ & 0.7500 & $-0.0147(8)$ & $0.0313(16)$ \\
C11 & $0.3828(14)$ & 0.7500 & $0.1960(8)$ & $0.0204(15)$ \\
C12 & $0.4932(10)$ & $0.6324(5)$ & $0.2559(6)$ & $0.0224(11)$ \\
C13 & $0.7107(10)$ & $0.6313(5)$ & $0.3688(6)$ & $0.0244(11)$ \\
H13 & 0.7842 & 0.5512 & 0.4091 & $0.029^{*}$ \\
C14 & $0.8200(14)$ & 0.7500 & $0.4224(8)$ & $0.0197(15)$ \\
C17 & $0.1523(16)$ & 0.7500 & $0.0799(9)$ & $0.0241(16)$ \\
\hline
\end{tabular}

Atomic displacement parameters $\left(\AA^{2}\right)$

\begin{tabular}{lllllll}
\hline & $U^{11}$ & $U^{22}$ & $U^{33}$ & $U^{12}$ & $U^{13}$ & $U^{23}$ \\
\hline Br12 & $0.0334(4)$ & $0.0160(3)$ & $0.0337(4)$ & $-0.0043(2)$ & $-0.0015(2)$ & $-0.0016(2)$ \\
Br14 & $0.0229(4)$ & $0.0239(4)$ & $0.0295(5)$ & 0.000 & $-0.0018(3)$ & 0.000 \\
N17 & $0.041(4)$ & $0.021(3)$ & $0.031(4)$ & 0.000 & $-0.006(3)$ & 0.000 \\
C11 & $0.022(3)$ & $0.025(4)$ & $0.015(4)$ & 0.000 & $0.005(3)$ & 0.000 \\
C12 & $0.023(2)$ & $0.016(2)$ & $0.029(3)$ & $-0.0012(19)$ & $0.006(2)$ & $0.001(2)$ \\
C13 & $0.024(2)$ & $0.017(3)$ & $0.033(3)$ & $0.004(2)$ & $0.007(2)$ & $0.004(2)$ \\
C14 & $0.024(3)$ & $0.025(4)$ & $0.011(3)$ & 0.000 & $0.003(3)$ & 0.000 \\
C17 & $0.030(4)$ & $0.011(3)$ & $0.032(4)$ & 0.000 & $0.004(3)$ & 0.000 \\
\hline
\end{tabular}

Geometric parameters $\left(\AA,{ }^{\circ}\right)$

\begin{tabular}{llll}
\hline $\mathrm{Br} 12-\mathrm{C} 12$ & $1.883(5)$ & $\mathrm{C} 12-\mathrm{C} 13$ & $1.380(8)$ \\
$\mathrm{Br} 14-\mathrm{C} 14$ & $1.881(7)$ & $\mathrm{C} 13-\mathrm{C} 14$ & $1.391(6)$ \\
$\mathrm{C} 11-\mathrm{C} 12$ & $1.401(6)$ & $\mathrm{C} 13-\mathrm{H} 13$ & 0.9500 \\
$\mathrm{C} 11-\mathrm{C} 17$ & $1.443(10)$ & $\mathrm{N} 17-\mathrm{C} 17$ & $1.144(10)$ \\
& & & \\
$\mathrm{C} 12-\mathrm{C} 11-\mathrm{C} 12^{\mathrm{i}}$ & $118.6(6)$ & $\mathrm{C} 12-\mathrm{C} 13-\mathrm{H} 13$ & 120.7 \\
$\mathrm{C} 12-\mathrm{C} 11-\mathrm{C} 17$ & $120.7(3)$ & $\mathrm{C} 14-\mathrm{C} 13-\mathrm{H} 13$ & 120.7 \\
$\mathrm{C} 13-\mathrm{C} 12-\mathrm{C} 11$ & $121.2(5)$ & $\mathrm{C} 13-\mathrm{C} 14-\mathrm{C} 13^{\mathrm{i}}$ & $121.9(6)$ \\
$\mathrm{C} 13-\mathrm{C} 12-\mathrm{Br} 12$ & $119.3(4)$ & $\mathrm{C} 13-\mathrm{C} 14-\mathrm{Br} 14$ & $178.4(9)$ \\
$\mathrm{C} 11-\mathrm{C} 12-\mathrm{Br} 12$ & $119.4(4)$ & $\mathrm{N} 17-\mathrm{C} 17-\mathrm{C} 11$ & \\
$\mathrm{C} 12-\mathrm{C} 13-\mathrm{C} 14$ & $118.6(5)$ & & $-0.2(10)$ \\
& & & $-177.8(5)$ \\
$\mathrm{C} 12 \mathrm{i}-\mathrm{C} 11-\mathrm{C} 12-\mathrm{C} 13$ & $-1.6(11)$ & $\mathrm{C} 11-\mathrm{C} 12-\mathrm{C} 13-\mathrm{C} 14$ & $2.0(12)$ \\
$\mathrm{C} 17-\mathrm{C} 11-\mathrm{C} 12-\mathrm{C} 13$ & $-178.9(7)$ & $\mathrm{Br} 12-\mathrm{C} 12-\mathrm{C} 13-\mathrm{C} 14$ & $179.2(5)$ \\
$\mathrm{C} 12-\mathrm{C} 11-\mathrm{C} 12-\mathrm{B} 12$ & $176.0(3)$ & $\mathrm{C} 12-\mathrm{C} 13-\mathrm{C} 14-\mathrm{C} 13^{\mathrm{i}}$ & \\
$\mathrm{C} 17-\mathrm{C} 11-\mathrm{C} 12-\mathrm{B} 12$ & $-1.3(9)$ & $\mathrm{C} 12-\mathrm{C} 13-\mathrm{C} 14-\mathrm{Br} 14$ & \\
& & & \\
\hline
\end{tabular}

Symmetry code: (i) $x,-y+3 / 2, z$. 
(RCN-II) 2,4,6-Tribromobenzonitrile - polytype II

Crystal data

$\mathrm{C}_{7} \mathrm{H}_{2} \mathrm{Br}_{3} \mathrm{~N}$

$M_{r}=339.83$

Orthorhombic, Pnma

Hall symbol: -P 2ac 2n

$a=13.6183(13) \AA$

$b=10.2147(10) \AA$

$c=12.4754(12) \AA$

$V=1735.4(3) \AA^{3}$

$Z=8$

Data collection

Bruker $1 \mathrm{~K}$ area-detector diffractometer

Radiation source: fine-focus sealed tube

Graphite monochromator

$\omega$ scans

Absorption correction: multi-scan

(SADABS; Bruker, 2002)

$T_{\min }=0.06, T_{\max }=0.37$

Refinement

Refinement on $F^{2}$

Least-squares matrix: full

$R\left[F^{2}>2 \sigma\left(F^{2}\right)\right]=0.028$

$w R\left(F^{2}\right)=0.063$

$S=1.02$

2093 reflections

115 parameters

0 restraints

Primary atom site location: structure-invariant direct methods
$F(000)=1248$

$D_{\mathrm{x}}=2.601 \mathrm{Mg} \mathrm{m}^{-3}$

Mo $K \alpha$ radiation, $\lambda=0.71073 \AA$

Cell parameters from 3180 reflections

$\theta=2.9-27.2^{\circ}$

$\mu=13.88 \mathrm{~mm}^{-1}$

$T=173 \mathrm{~K}$

Plate, colorless

$0.25 \times 0.20 \times 0.07 \mathrm{~mm}$

16607 measured reflections

2093 independent reflections

1692 reflections with $I>2 \sigma(I)$

$R_{\text {int }}=0.052$

$\theta_{\max }=27.5^{\circ}, \theta_{\min }=2.2^{\circ}$

$h=-17 \rightarrow 17$

$k=-13 \rightarrow 13$

$l=-16 \rightarrow 16$

Secondary atom site location: difference Fourier map

Hydrogen site location: inferred from neighbouring sites

$\mathrm{H}$-atom parameters constrained

$w=1 /\left[\sigma^{2}\left(F_{\mathrm{o}}^{2}\right)+(0.030 P)^{2}+1.560 P\right]$

where $P=\left(F_{\mathrm{o}}^{2}+2 F_{\mathrm{c}}{ }^{2}\right) / 3$

$(\Delta / \sigma)_{\max }=0.001$

$\Delta \rho_{\max }=0.44 \mathrm{e} \AA^{-3}$

$\Delta \rho_{\min }=-0.69$ e $\AA^{-3}$

Fractional atomic coordinates and isotropic or equivalent isotropic displacement parameters $\left(\hat{A}^{2}\right)$

\begin{tabular}{lllll}
\hline & $x$ & $y$ & $z$ & $U_{\text {iso }} * / U_{\text {eq }}$ \\
\hline Br22 & $0.13341(3)$ & $0.52761(3)$ & $0.04244(3)$ & $0.02658(11)$ \\
$\mathrm{Br} 24$ & $0.14558(4)$ & 0.2500 & $0.43375(4)$ & $0.02477(13)$ \\
$\mathrm{C} 21$ & $0.1318(3)$ & 0.2500 & $0.0608(4)$ & $0.0197(10)$ \\
$\mathrm{C} 22$ & $0.1359(2)$ & $0.3683(3)$ & $0.1174(3)$ & $0.0206(7)$ \\
$\mathrm{C} 23$ & $0.1418(2)$ & $0.3697(3)$ & $0.2282(3)$ & $0.0217(7)$ \\
$\mathrm{H} 23$ & 0.1444 & 0.4500 & 0.2666 & $0.026^{*}$ \\
$\mathrm{C} 24$ & $0.1437(3)$ & 0.2500 & $0.2821(4)$ & $0.0190(10)$ \\
$\mathrm{C} 27$ & $0.1207(4)$ & 0.2500 & $-0.0545(4)$ & $0.0257(11)$ \\
$\mathrm{N} 27$ & $0.1115(3)$ & 0.2500 & $-0.1447(4)$ & $0.0332(11)$ \\
$\mathrm{Br} 32$ & $0.10699(3)$ & $0.47273(3)$ & $0.69146(3)$ & $0.02650(11)$ \\
$\mathrm{Br} 34$ & $0.12804(4)$ & 0.7500 & $0.29979(4)$ & $0.02786(13)$ \\
$\mathrm{C} 31$ & $0.1095(3)$ & 0.7500 & $0.6720(3)$ & $0.0175(9)$ \\
$\mathrm{C} 32$ & $0.1116(2)$ & $0.6320(3)$ & $0.6155(3)$ & $0.0195(7)$ \\
$\mathrm{C} 33$ & $0.1171(2)$ & $0.6315(3)$ & $0.5049(3)$ & $0.0201(7)$
\end{tabular}




\begin{tabular}{lllll}
$\mathrm{H} 33$ & 0.1189 & 0.5511 & 0.4666 & $0.024^{*}$ \\
$\mathrm{C} 34$ & $0.1199(3)$ & 0.7500 & $0.4508(4)$ & $0.0196(10)$ \\
$\mathrm{C} 37$ & $0.1056(3)$ & 0.7500 & $0.7873(4)$ & $0.0200(10)$ \\
N37 & $0.1015(3)$ & 0.7500 & $0.8798(3)$ & $0.0255(9)$ \\
\hline
\end{tabular}

Atomic displacement parameters $\left(\AA^{2}\right)$

\begin{tabular}{lllllll}
\hline & $U^{11}$ & $U^{22}$ & $U^{33}$ & $U^{12}$ & $U^{13}$ & $U^{23}$ \\
\hline $\mathrm{Br} 22$ & $0.0364(2)$ & $0.01742(19)$ & $0.0259(2)$ & $-0.00058(15)$ & $-0.00196(15)$ & $0.00451(14)$ \\
$\mathrm{Br} 24$ & $0.0303(3)$ & $0.0261(3)$ & $0.0179(2)$ & 0.000 & $0.00098(19)$ & 0.000 \\
$\mathrm{C} 21$ & $0.018(2)$ & $0.021(3)$ & $0.021(2)$ & 0.000 & $0.0017(19)$ & 0.000 \\
$\mathrm{C} 22$ & $0.0208(16)$ & $0.0182(16)$ & $0.0227(17)$ & $0.0016(14)$ & $-0.0003(13)$ & $0.0017(14)$ \\
$\mathrm{C} 23$ & $0.0225(16)$ & $0.0186(18)$ & $0.0240(17)$ & $-0.0012(14)$ & $-0.0012(14)$ & $-0.0017(14)$ \\
$\mathrm{C} 24$ & $0.021(2)$ & $0.021(3)$ & $0.015(2)$ & 0.000 & $0.0022(18)$ & 0.000 \\
$\mathrm{C} 27$ & $0.028(3)$ & $0.020(3)$ & $0.029(3)$ & 0.000 & $0.000(2)$ & 0.000 \\
$\mathrm{~N} 27$ & $0.048(3)$ & $0.026(2)$ & $0.026(3)$ & 0.000 & $-0.002(2)$ & 0.000 \\
$\mathrm{Br} 32$ & $0.0386(2)$ & $0.01618(19)$ & $0.02475(19)$ & $-0.00117(15)$ & $0.00205(14)$ & $0.00374(14)$ \\
$\mathrm{Br} 34$ & $0.0418(3)$ & $0.0243(3)$ & $0.0174(2)$ & 0.000 & $-0.0003(2)$ & 0.000 \\
$\mathrm{C} 31$ & $0.017(2)$ & $0.021(2)$ & $0.015(2)$ & 0.000 & $-0.0003(17)$ & 0.000 \\
$\mathrm{C} 32$ & $0.0182(15)$ & $0.0163(16)$ & $0.0241(17)$ & $0.0004(13)$ & $-0.0006(13)$ & $0.0044(14)$ \\
$\mathrm{C} 33$ & $0.0229(17)$ & $0.0157(18)$ & $0.0216(17)$ & $0.0015(14)$ & $-0.0009(13)$ & $-0.0018(14)$ \\
$\mathrm{C} 34$ & $0.025(2)$ & $0.018(2)$ & $0.015(2)$ & 0.000 & $-0.0001(18)$ & 0.000 \\
$\mathrm{C} 37$ & $0.023(2)$ & $0.014(2)$ & $0.023(3)$ & 0.000 & $-0.0009(19)$ & 0.000 \\
$\mathrm{~N} 37$ & $0.030(2)$ & $0.024(2)$ & $0.023(2)$ & 0.000 & $-0.0002(17)$ & 0.000 \\
& & & & & & \\
\hline
\end{tabular}

Geometric parameters $\left(\AA,{ }^{\circ}\right)$

\begin{tabular}{llll}
\hline $\mathrm{Br} 22-\mathrm{C} 22$ & $1.877(3)$ & $\mathrm{Br} 32-\mathrm{C} 32$ & $1.884(3)$ \\
$\mathrm{Br} 24-\mathrm{C} 24$ & $1.892(5)$ & $\mathrm{Br} 3-\mathrm{C} 34$ & $1.887(4)$ \\
$\mathrm{C} 21-\mathrm{C} 22$ & $1.400(4)$ & $\mathrm{C} 31-\mathrm{C} 32$ & $1.396(4)$ \\
$\mathrm{C} 21-\mathrm{C} 27$ & $1.446(7)$ & $\mathrm{C} 31-\mathrm{C} 37$ & $1.439(6)$ \\
$\mathrm{C} 22-\mathrm{C} 23$ & $1.385(5)$ & $\mathrm{C} 32-\mathrm{C} 33$ & $1.382(5)$ \\
$\mathrm{C} 23-\mathrm{C} 24$ & $1.395(4)$ & $\mathrm{C} 33-\mathrm{C} 34$ & $1.387(4)$ \\
$\mathrm{C} 23-\mathrm{H} 23$ & 0.9500 & $\mathrm{C} 33-\mathrm{H} 33$ & 0.9500 \\
$\mathrm{C} 27-\mathrm{N} 27$ & $1.132(7)$ & $\mathrm{C} 37-\mathrm{N} 37$ & $1.156(6)$ \\
& & & $119.3(4)$ \\
$\mathrm{C} 22-\mathrm{C} 21-\mathrm{C} 22$ & $119.3(4)$ & $\mathrm{C} 32 \mathrm{ii}-\mathrm{C} 31-\mathrm{C} 32$ & $120.3(2)$ \\
$\mathrm{C} 22-\mathrm{C} 21-\mathrm{C} 27$ & $120.3(2)$ & $\mathrm{C} 32-\mathrm{C} 31-\mathrm{C} 37$ & $120.6(3)$ \\
$\mathrm{C} 23-\mathrm{C} 22-\mathrm{C} 21$ & $120.9(3)$ & $\mathrm{C} 33-\mathrm{C} 32-\mathrm{C} 31$ & $119.4(2)$ \\
$\mathrm{C} 23-\mathrm{C} 22-\mathrm{Br} 22$ & $119.3(3)$ & $\mathrm{C} 33-\mathrm{C} 32-\mathrm{Br} 32$ & $118.9(3)$ \\
$\mathrm{C} 21-\mathrm{C} 22-\mathrm{Br} 22$ & $119.8(3)$ & $\mathrm{C} 31-\mathrm{C} 32-\mathrm{B} 32$ & 120.5 \\
$\mathrm{C} 22-\mathrm{C} 23-\mathrm{C} 24$ & $118.2(3)$ & $\mathrm{C} 32-\mathrm{C} 33-\mathrm{C} 34$ & 120.5 \\
$\mathrm{C} 22-\mathrm{C} 23-\mathrm{H} 23$ & 120.9 & $\mathrm{C} 32-\mathrm{C} 33-\mathrm{H} 33$ & $121.6(4)$ \\
$\mathrm{C} 24-\mathrm{C} 23-\mathrm{H} 23$ & 120.9 & $\mathrm{C} 34-\mathrm{C} 33-\mathrm{H} 33$ & $119.2(2)$ \\
$\mathrm{C} 23-\mathrm{C} 24-\mathrm{C} 23$ & $122.4(4)$ & $\mathrm{C} 33-\mathrm{C} 34-\mathrm{C} 33$ & $179.3(5)$ \\
$\mathrm{C} 23-\mathrm{C} 24-\mathrm{Br} 24$ & $118.8(2)$ & $\mathrm{C} 33-\mathrm{C} 34-\mathrm{B} 34$ & \\
$\mathrm{~N} 27-\mathrm{C} 27-\mathrm{C} 21$ & $179.7(5)$ & $\mathrm{N} 37-\mathrm{C} 37-\mathrm{C} 31$ &
\end{tabular}




$\begin{array}{llll}\mathrm{C} 22^{\mathrm{i}}-\mathrm{C} 21-\mathrm{C} 22-\mathrm{C} 23 & -1.4(6) & \mathrm{C} 32^{\mathrm{ii}}-\mathrm{C} 31-\mathrm{C} 32-\mathrm{C} 33 & 0.8(6) \\ \mathrm{C} 27-\mathrm{C} 21-\mathrm{C} 22-\mathrm{C} 23 & 176.8(4) & \mathrm{C} 37-\mathrm{C} 31-\mathrm{C} 32-\mathrm{C} 33 & -178.9(4) \\ \mathrm{C} 22^{\mathrm{i}}-\mathrm{C} 21-\mathrm{C} 22-\mathrm{Br} 22 & 178.6(2) & \mathrm{C} 32^{\mathrm{i}}-\mathrm{C} 31-\mathrm{C} 32-\mathrm{Br} 32 & -179.2(2) \\ \mathrm{C} 27-\mathrm{C} 21-\mathrm{C} 22-\mathrm{Br} 22 & -3.2(5) & \mathrm{C} 37-\mathrm{C} 31-\mathrm{C} 32-\mathrm{Br} 32 & 1.1(5) \\ \mathrm{C} 21-\mathrm{C} 22-\mathrm{C} 23-\mathrm{C} 24 & 0.1(5) & \mathrm{C} 31-\mathrm{C} 32-\mathrm{C} 33-\mathrm{C} 34 & -0.3(5) \\ \mathrm{Br} 22-\mathrm{C} 22-\mathrm{C} 23-\mathrm{C} 24 & -179.9(3) & \mathrm{B} 32-\mathrm{C} 32-\mathrm{C} 33-\mathrm{C} 34 & 179.7(3) \\ \mathrm{C} 22-\mathrm{C} 23-\mathrm{C} 24-\mathrm{C} 23^{\mathrm{i}} & 1.3(7) & \mathrm{C} 32-\mathrm{C} 33-\mathrm{C} 34-\mathrm{C} 33^{\mathrm{ii}} & -0.2(7) \\ \mathrm{C} 22-\mathrm{C} 23-\mathrm{C} 24-\mathrm{Br} 24 & -177.1(2) & \mathrm{C} 32-\mathrm{C} 33-\mathrm{C} 34-\mathrm{Br} 34 & 179.7(2)\end{array}$

Symmetry codes: (i) $x,-y+1 / 2, z$; (ii) $x,-y+3 / 2, z$.

(RCN-III) 2,4,6-Tribromobenzonitrile - polytype III

Crystal data

$\mathrm{C}_{7} \mathrm{H}_{2} \mathrm{Br}_{3} \mathrm{~N}$

$M_{r}=339.83$

Orthorhombic, Pnma

Hall symbol: -P 2ac $2 \mathrm{n}$

$a=20.399$ (4) $\AA$

$b=10.2167(18) \AA$

$c=12.493(2) \AA$

$V=2603.7(8) \AA^{3}$

$Z=12$

$F(000)=1872$

$D_{\mathrm{x}}=2.601 \mathrm{Mg} \mathrm{m}^{-3}$

Mo $K \alpha$ radiation, $\lambda=0.71073 \AA$

Cell parameters from 2928 reflections

$\theta=2.6-26.7^{\circ}$

$\mu=13.87 \mathrm{~mm}^{-1}$

$T=173 \mathrm{~K}$

Needle, colorless

$0.50 \times 0.15 \times 0.10 \mathrm{~mm}$

\section{Data collection}

Bruker 1K area-detector diffractometer

Radiation source: fine-focus sealed tube

Graphite monochromator

$\omega$ scans

Absorption correction: multi-scan

(SADABS; Bruker, 2002)

$T_{\min }=0.054, T_{\max }=0.337$

\section{Refinement}

Refinement on $F^{2}$

Least-squares matrix: full

$R\left[F^{2}>2 \sigma\left(F^{2}\right)\right]=0.023$

$w R\left(F^{2}\right)=0.046$

$S=1.07$

2691 reflections

173 parameters

0 restraints

Primary atom site location: structure-invariant direct methods

Secondary atom site location: difference Fourier map

Fractional atomic coordinates and isotropic or equivalent isotropic displacement parameters $\left(\hat{A}^{2}\right)$

\begin{tabular}{lllll}
\hline & $x$ & $y$ & $z$ & $U_{\text {iso }} * / U_{\text {eq }}$ \\
\hline $\mathrm{Br} 42$ & $0.340999(16)$ & $0.52705(3)$ & $-0.05464(3)$ & $0.02707(10)$ \\
$\mathrm{Br} 44$ & $0.32895(2)$ & 0.2500 & $0.33679(4)$ & $0.02913(13)$
\end{tabular}

22804 measured reflections

2691 independent reflections

2165 reflections with $I>2 \sigma(I)$

$R_{\text {int }}=0.055$

$\theta_{\max }=26.0^{\circ}, \theta_{\min }=2.6^{\circ}$

$k=-12 \rightarrow 12$

$l=-15 \rightarrow 15$

Hydrogen site location: inferred from neighbouring sites

$\mathrm{H}$-atom parameters constrained

$w=1 /\left[\sigma^{2}\left(F_{\mathrm{o}}^{2}\right)+(0.0096 P)^{2}+3.390 P\right]$

where $P=\left(F_{\mathrm{o}}^{2}+2 F_{\mathrm{c}}^{2}\right) / 3$

$(\Delta / \sigma)_{\max }=0.001$

$\Delta \rho_{\max }=0.56 \mathrm{e} \AA^{-3}$

$\Delta \rho_{\min }=-0.49$ e $\AA^{-3}$

Extinction correction: SHELXL, $\mathrm{Fc}^{*}=\mathrm{kFc}\left[1+0.001 \mathrm{xFc}^{2} \lambda^{3} / \sin (2 \theta)\right]^{-1 / 4}$

Extinction coefficient: 0.00028 (3) $h=-24 \rightarrow 24$ 


$\begin{array}{lllll}\text { Br52 } & 0.332551(16) & 0.47245(3) & 0.59427(3) & 0.02775(9) \\ \text { Br54 } & 0.32011(2) & 0.7500 & 0.20370(3) & 0.02542(12) \\ \text { Br62 } & 0.511839(16) & 0.52774(3) & 0.67598(3) & 0.02743(10) \\ \text { Br64 } & 0.50730(2) & 0.2500 & 1.06666(4) & 0.02435(12) \\ \text { C41 } & 0.33919(19) & 0.2500 & -0.0353(3) & 0.0182(9) \\ \text { C42 } & 0.33772(14) & 0.3675(3) & 0.0214(2) & 0.0207(7) \\ \text { C43 } & 0.33432(14) & 0.3686(3) & 0.1321(2) & 0.0226(7) \\ \text { H43 } & 0.3331 & 0.4487 & 0.1706 & 0.027^{*} \\ \text { C44 } & 0.3328(2) & 0.2500 & 0.1851(4) & 0.0219(10) \\ \text { C47 } & 0.3440(2) & 0.2500 & -0.1508(4) & 0.0218(10) \\ \text { N47 } & 0.34814(18) & 0.2500 & -0.2423(3) & 0.0272(9) \\ \text { C51 } & 0.3338(2) & 0.7500 & 0.5758(4) & 0.0221(10) \\ \text { C52 } & 0.33096(14) & 0.6320(3) & 0.5193(2) & 0.0211(7) \\ \text { C53 } & 0.32641(14) & 0.6314(3) & 0.4085(2) & 0.0228(7) \\ \text { H53 } & 0.3246 & 0.5512 & 0.3701 & 0.027^{*} \\ \text { C54 } & 0.3245(2) & 0.7500 & 0.3549(3) & 0.0204(10) \\ \text { C57 } & 0.3399(2) & 0.7500 & 0.6908(4) & 0.0225(10) \\ \text { N57 } & 0.3445(2) & 0.7500 & 0.7823(3) & 0.0329(10) \\ \text { C61 } & 0.5080(2) & 0.2500 & 0.6942(4) & 0.0204(10) \\ \text { C62 } & 0.50889(14) & 0.3676(3) & 0.7509(2) & 0.0216(7) \\ \text { C63 } & 0.50886(14) & 0.3686(3) & 0.8618(2) & 0.0218(7) \\ \text { H63 } & 0.5092 & 0.4488 & 0.9002 & 0.026^{*} \\ \text { C64 } & 0.5083(2) & 0.2500 & 0.9155(4) & 0.0200(10) \\ \text { C67 } & 0.5049(2) & 0.2500 & 0.5783(4) & 0.0225(10) \\ \text { N67 } & 0.5024(2) & 0.2500 & 0.4872(3) & 0.0329(10) \\ & & & & \end{array}$

Atomic displacement parameters $\left(A^{2}\right)$

\begin{tabular}{lllllll}
\hline & $U^{11}$ & $U^{22}$ & $U^{33}$ & $U^{12}$ & $U^{13}$ & $U^{23}$ \\
\hline Br42 & $0.03864(19)$ & $0.01711(17)$ & $0.02545(18)$ & $-0.00178(14)$ & $0.00143(15)$ & $0.00391(15)$ \\
Br44 & $0.0432(3)$ & $0.0252(3)$ & $0.0190(3)$ & 0.000 & $0.0007(2)$ & 0.000 \\
Br52 & $0.03826(19)$ & $0.01818(17)$ & $0.02682(18)$ & $-0.00040(15)$ & $-0.00267(15)$ & $0.00467(15)$ \\
Br54 & $0.0309(3)$ & $0.0270(3)$ & $0.0184(2)$ & 0.000 & $0.0017(2)$ & 0.000 \\
Br62 & $0.03877(19)$ & $0.01736(17)$ & $0.02617(18)$ & $-0.00080(15)$ & $-0.00036(15)$ & $0.00376(15)$ \\
Br64 & $0.0300(2)$ & $0.0243(3)$ & $0.0188(2)$ & 0.000 & $-0.00018(19)$ & 0.000 \\
C41 & $0.016(2)$ & $0.017(2)$ & $0.022(2)$ & 0.000 & $-0.0010(18)$ & 0.000 \\
C42 & $0.0214(15)$ & $0.0175(17)$ & $0.0233(17)$ & $-0.0001(14)$ & $-0.0011(13)$ & $0.0052(14)$ \\
C43 & $0.0269(16)$ & $0.0160(17)$ & $0.0248(17)$ & $-0.0016(14)$ & $-0.0003(14)$ & $-0.0016(15)$ \\
C44 & $0.023(2)$ & $0.024(3)$ & $0.019(2)$ & 0.000 & $0.0000(19)$ & 0.000 \\
C47 & $0.020(2)$ & $0.016(2)$ & $0.029(3)$ & 0.000 & $-0.004(2)$ & 0.000 \\
N47 & $0.033(2)$ & $0.022(2)$ & $0.026(2)$ & 0.000 & $-0.0017(19)$ & 0.000 \\
C51 & $0.016(2)$ & $0.026(3)$ & $0.024(2)$ & 0.000 & $0.001(2)$ & 0.000 \\
C52 & $0.0244(15)$ & $0.0154(17)$ & $0.0234(16)$ & $0.0002(14)$ & $-0.0002(13)$ & $0.0037(14)$ \\
C53 & $0.0255(16)$ & $0.0198(18)$ & $0.0232(17)$ & $0.0025(14)$ & $0.0024(14)$ & $-0.0031(15)$ \\
C54 & $0.020(2)$ & $0.024(3)$ & $0.017(2)$ & 0.000 & $0.0021(18)$ & 0.000 \\
C57 & $0.025(2)$ & $0.015(2)$ & $0.027(3)$ & 0.000 & $-0.003(2)$ & 0.000 \\
N57 & $0.048(3)$ & $0.027(2)$ & $0.024(2)$ & 0.000 & $-0.001(2)$ & 0.000 \\
C61 & $0.020(2)$ & $0.022(2)$ & $0.020(2)$ & 0.000 & $0.0041(19)$ & 0.000
\end{tabular}




\begin{tabular}{lllllll} 
C62 & $0.0199(15)$ & $0.0188(17)$ & $0.0261(17)$ & $0.0017(13)$ & $0.0011(13)$ & $0.0047(15)$ \\
C63 & $0.0236(16)$ & $0.0178(18)$ & $0.0239(16)$ & $0.0007(14)$ & $0.0018(13)$ & $-0.0028(15)$ \\
C64 & $0.020(2)$ & $0.022(2)$ & $0.018(2)$ & 0.000 & $0.0017(19)$ & 0.000 \\
C67 & $0.028(2)$ & $0.016(2)$ & $0.024(3)$ & 0.000 & $0.000(2)$ & 0.000 \\
N67 & $0.055(3)$ & $0.021(2)$ & $0.024(2)$ & 0.000 & $-0.002(2)$ & 0.000 \\
\hline
\end{tabular}

Geometric parameters $\left(\hat{A},{ }^{\circ}\right)$

\begin{tabular}{|c|c|c|c|}
\hline $\mathrm{Br} 42-\mathrm{C} 42$ & $1.888(3)$ & $\mathrm{C} 51-\mathrm{C} 52$ & $1.399(4)$ \\
\hline $\mathrm{Br} 44-\mathrm{C} 44$ & $1.897(5)$ & $\mathrm{C} 51-\mathrm{C} 57$ & $1.443(6)$ \\
\hline $\mathrm{Br} 52-\mathrm{C} 52$ & $1.880(3)$ & $\mathrm{C} 52-\mathrm{C} 53$ & $1.387(4)$ \\
\hline $\mathrm{Br} 54-\mathrm{C} 54$ & $1.892(4)$ & $\mathrm{C} 53-\mathrm{C} 54$ & $1.384(4)$ \\
\hline $\mathrm{Br} 62-\mathrm{C} 62$ & $1.885(3)$ & $\mathrm{C} 53-\mathrm{H} 53$ & 0.9500 \\
\hline Br64-C64 & $1.889(4)$ & $\mathrm{C} 57-\mathrm{N} 57$ & $1.147(6)$ \\
\hline $\mathrm{C} 41-\mathrm{C} 42$ & $1.394(4)$ & $\mathrm{C} 61-\mathrm{C} 62$ & $1.395(4)$ \\
\hline $\mathrm{C} 41-\mathrm{C} 47$ & $1.447(6)$ & $\mathrm{C} 61-\mathrm{C} 67$ & $1.450(6)$ \\
\hline $\mathrm{C} 42-\mathrm{C} 43$ & $1.384(4)$ & $\mathrm{C} 62-\mathrm{C} 63$ & $1.386(4)$ \\
\hline $\mathrm{C} 43-\mathrm{C} 44$ & $1.381(4)$ & $\mathrm{C} 63-\mathrm{C} 64$ & $1.385(4)$ \\
\hline $\mathrm{C} 43-\mathrm{H} 43$ & 0.9500 & C63-H63 & 0.9500 \\
\hline $\mathrm{C} 47-\mathrm{N} 47$ & $1.146(6)$ & C67-N67 & $1.139(6)$ \\
\hline $\mathrm{C} 42-\mathrm{C} 41-\mathrm{C} 42^{\mathrm{i}}$ & $118.9(4)$ & $\mathrm{C} 54-\mathrm{C} 53-\mathrm{H} 53$ & 120.6 \\
\hline $\mathrm{C} 42-\mathrm{C} 41-\mathrm{C} 47$ & $120.5(2)$ & $\mathrm{C} 52-\mathrm{C} 53-\mathrm{H} 53$ & 120.6 \\
\hline $\mathrm{C} 43-\mathrm{C} 42-\mathrm{C} 41$ & $121.0(3)$ & $\mathrm{C} 53^{\mathrm{ii}}-\mathrm{C} 54-\mathrm{C} 53$ & $122.1(4)$ \\
\hline $\mathrm{C} 43-\mathrm{C} 42-\mathrm{Br} 42$ & $119.9(2)$ & $\mathrm{C} 53-\mathrm{C} 54-\mathrm{Br} 54$ & $119.0(2)$ \\
\hline $\mathrm{C} 41-\mathrm{C} 42-\mathrm{Br} 42$ & $119.2(2)$ & $\mathrm{N} 57-\mathrm{C} 57-\mathrm{C} 51$ & $179.8(5)$ \\
\hline $\mathrm{C} 44-\mathrm{C} 43-\mathrm{C} 42$ & $118.3(3)$ & $\mathrm{C} 62^{\mathrm{i}}-\mathrm{C} 61-\mathrm{C} 62$ & $119.0(4)$ \\
\hline $\mathrm{C} 44-\mathrm{C} 43-\mathrm{H} 43$ & 120.9 & $\mathrm{C} 62-\mathrm{C} 61-\mathrm{C} 67$ & $120.5(2)$ \\
\hline $\mathrm{C} 42-\mathrm{C} 43-\mathrm{H} 43$ & 120.9 & $\mathrm{C} 63-\mathrm{C} 62-\mathrm{C} 61$ & $120.9(3)$ \\
\hline 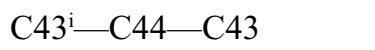 & $122.6(4)$ & $\mathrm{C} 63-\mathrm{C} 62-\mathrm{Br} 62$ & $119.4(3)$ \\
\hline $\mathrm{C} 43-\mathrm{C} 44-\mathrm{Br} 44$ & $118.7(2)$ & $\mathrm{C} 61-\mathrm{C} 62-\mathrm{Br} 62$ & $119.8(2)$ \\
\hline $\mathrm{N} 47-\mathrm{C} 47-\mathrm{C} 41$ & $179.7(5)$ & $\mathrm{C} 64-\mathrm{C} 63-\mathrm{C} 62$ & $118.6(3)$ \\
\hline $\mathrm{C} 52^{\mathrm{ii}}-\mathrm{C} 51-\mathrm{C} 52$ & $119.1(4)$ & $\mathrm{C} 64-\mathrm{C} 63-\mathrm{H} 63$ & 120.7 \\
\hline $\mathrm{C} 52-\mathrm{C} 51-\mathrm{C} 57$ & $120.4(2)$ & $\mathrm{C} 62-\mathrm{C} 63-\mathrm{H} 63$ & 120.7 \\
\hline $\mathrm{C} 53-\mathrm{C} 52-\mathrm{C} 51$ & $120.7(3)$ & 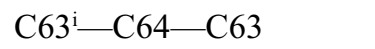 & $122.0(4)$ \\
\hline $\mathrm{C} 53-\mathrm{C} 52-\mathrm{Br} 52$ & $119.7(2)$ & $\mathrm{C} 63-\mathrm{C} 64-\mathrm{Br} 64$ & $119.0(2)$ \\
\hline $\mathrm{C} 51-\mathrm{C} 52-\mathrm{Br} 52$ & $119.7(2)$ & N67-C67-C61 & $180.0(5)$ \\
\hline $\mathrm{C} 54-\mathrm{C} 53-\mathrm{C} 52$ & $118.7(3)$ & & \\
\hline $\mathrm{C} 42^{\mathrm{i}}-\mathrm{C} 41-\mathrm{C} 42-\mathrm{C} 43$ & $-0.5(6)$ & $\mathrm{C} 51-\mathrm{C} 52-\mathrm{C} 53-\mathrm{C} 54$ & $-0.2(5)$ \\
\hline $\mathrm{C} 47-\mathrm{C} 41-\mathrm{C} 42-\mathrm{C} 43$ & $-178.8(3)$ & $\mathrm{Br} 52-\mathrm{C} 52-\mathrm{C} 53-\mathrm{C} 54$ & $179.2(3)$ \\
\hline $\mathrm{C} 42^{\mathrm{i}}-\mathrm{C} 41-\mathrm{C} 42-\mathrm{Br} 42$ & $179.07(19)$ & $\mathrm{C} 52-\mathrm{C} 53-\mathrm{C} 54-\mathrm{C} 53^{\mathrm{ii}}$ & $-0.6(6)$ \\
\hline $\mathrm{C} 47-\mathrm{C} 41-\mathrm{C} 42-\mathrm{Br} 42$ & $0.8(5)$ & $\mathrm{C} 52-\mathrm{C} 53-\mathrm{C} 54-\mathrm{Br} 54$ & $178.7(2)$ \\
\hline $\mathrm{C} 41-\mathrm{C} 42-\mathrm{C} 43-\mathrm{C} 44$ & $0.4(5)$ & $\mathrm{C} 62^{\mathrm{i}}-\mathrm{C} 61-\mathrm{C} 62-\mathrm{C} 63$ & $-1.7(6)$ \\
\hline $\mathrm{Br} 42-\mathrm{C} 42-\mathrm{C} 43-\mathrm{C} 44$ & $-179.2(3)$ & $\mathrm{C} 67-\mathrm{C} 61-\mathrm{C} 62-\mathrm{C} 63$ & $177.0(3)$ \\
\hline $\mathrm{C} 42-\mathrm{C} 43-\mathrm{C} 44-\mathrm{C} 43^{\mathrm{i}}$ & $-0.2(6)$ & $\mathrm{C} 62^{\mathrm{i}}-\mathrm{C} 61-\mathrm{C} 62-\mathrm{Br} 62$ & $177.1(2)$ \\
\hline $\mathrm{C} 42-\mathrm{C} 43-\mathrm{C} 44-\mathrm{Br} 44$ & $179.4(2)$ & $\mathrm{C} 67-\mathrm{C} 61-\mathrm{C} 62-\mathrm{Br} 62$ & $-4.2(5)$ \\
\hline $\mathrm{C} 52^{\mathrm{ii}}-\mathrm{C} 51-\mathrm{C} 52-\mathrm{C} 53$ & $0.9(6)$ & $\mathrm{C} 61-\mathrm{C} 62-\mathrm{C} 63-\mathrm{C} 64$ & $0.4(5)$ \\
\hline
\end{tabular}




$\begin{array}{llll}\mathrm{C} 57-\mathrm{C} 51-\mathrm{C} 52-\mathrm{C} 53 & -178.7(3) & \mathrm{Br} 62-\mathrm{C} 62-\mathrm{C} 63-\mathrm{C} 64 & -178.4(3) \\ \mathrm{C} 52^{\mathrm{ii}}-\mathrm{C} 51-\mathrm{C} 52-\mathrm{Br} 52 & -178.48(19) & \mathrm{C} 62-\mathrm{C} 63-\mathrm{C} 64-\mathrm{C} 63^{\mathrm{i}} & 1.0(6) \\ \mathrm{C} 57-\mathrm{C} 51-\mathrm{C} 52-\mathrm{Br} 52 & 1.9(5) & \mathrm{C} 62-\mathrm{C} 63-\mathrm{C} 64-\mathrm{Br} 64 & -179.3(2)\end{array}$

Symmetry codes: (i) $x,-y+1 / 2, z$; (ii) $x,-y+3 / 2, z$.

(RNC-II) 1,3,5-Tribromo-2-isocyanobenzene - polytype II

Crystal data

$\mathrm{C}_{7} \mathrm{H}_{2} \mathrm{Br}_{3} \mathrm{~N}$

$M_{r}=339.83$

Orthorhombic, Pnma

$a=13.5916(18) \AA$

$b=10.1464(13) \AA$

$c=12.6158(16) \AA$

$V=1739.8(4) \AA^{3}$

$Z=8$

$F(000)=1248$

Data collection

Bruker APEXII CCD

diffractometer

Radiation source: sealed tube

$\varphi$ and $\omega$ scans

Absorption correction: multi-scan

(SADABS; Bruker, 2002)

$T_{\min }=0.170, T_{\max }=0.333$

19459 measured reflections

\section{Refinement}

Refinement on $F^{2}$

Least-squares matrix: full

$R\left[F^{2}>2 \sigma\left(F^{2}\right)\right]=0.025$

$w R\left(F^{2}\right)=0.055$

$S=1.06$

2105 reflections

116 parameters

0 restraints

Hydrogen site location: inferred from neighbouring sites
$D_{\mathrm{x}}=2.595 \mathrm{Mg} \mathrm{m}^{-3}$

Mo $K \alpha$ radiation, $\lambda=0.71073 \AA$

Cell parameters from 2721 reflections

$\theta=3.0-27.4^{\circ}$

$\mu=13.84 \mathrm{~mm}^{-1}$

$T=173 \mathrm{~K}$

Block, colourless

$0.40 \times 0.35 \times 0.20 \mathrm{~mm}$

2105 independent reflections

1638 reflections with $I>2 \sigma(I)$

$R_{\text {int }}=0.078$

$\theta_{\max }=27.5^{\circ}, \theta_{\min }=2.2^{\circ}$

$h=-17 \rightarrow 17$

$k=-13 \rightarrow 13$

$l=-16 \rightarrow 16$

$\mathrm{H}$-atom parameters constrained

$w=1 /\left[\sigma^{2}\left(F_{\mathrm{o}}{ }^{2}\right)+(0.0121 P)^{2}+1.0004 P\right]$ where $P=\left(F_{\mathrm{o}}^{2}+2 F_{\mathrm{c}}{ }^{2}\right) / 3$

$(\Delta / \sigma)_{\max }=0.001$

$\Delta \rho_{\max }=0.44$ e $\AA^{-3}$

$\Delta \rho_{\min }=-0.48$ e $\AA^{-3}$

Extinction correction: SHELXL, $\mathrm{Fc}^{*}=\mathrm{kFc}\left[1+0.001 \times \mathrm{Fc}^{2} \lambda^{3} / \sin (2 \theta)\right]^{-1 / 4}$

Extinction coefficient: 0.00269 (12)

\section{Special details}

Geometry. All esds (except the esd in the dihedral angle between two 1.s. planes) are estimated using the full covariance matrix. The cell esds are taken into account individually in the estimation of esds in distances, angles and torsion angles; correlations between esds in cell parameters are only used when they are defined by crystal symmetry. An approximate (isotropic) treatment of cell esds is used for estimating esds involving 1.s. planes.

Fractional atomic coordinates and isotropic or equivalent isotropic displacement parameters $\left(\AA^{2}\right)$

\begin{tabular}{lllll}
\hline & $x$ & $y$ & $z$ & $U_{\text {iso }} * / U_{\text {eq }}$ \\
\hline C121 & $0.3678(3)$ & 0.7500 & $0.5680(3)$ & $0.0162(9)$ \\
C122 & $0.3637(2)$ & $0.6315(3)$ & $0.6238(2)$ & $0.0176(7)$ \\
C123 & $0.3573(2)$ & $0.6306(3)$ & $0.7332(2)$ & $0.0182(7)$ \\
H123 & 0.3541 & 0.5499 & 0.7712 & $0.022^{*}$
\end{tabular}




$\begin{array}{lllll}\mathrm{C} 124 & 0.3559(3) & 0.7500 & 0.7858(4) & 0.0173(9) \\ \mathrm{N} 127 & 0.3793(3) & 0.7500 & 0.4583(3) & 0.0215(9) \\ \mathrm{C} 127 & 0.3909(4) & 0.7500 & 0.3682(4) & 0.0285(12) \\ \mathrm{B} 122 & 0.36763(3) & 0.47074(3) & 0.54952(3) & 0.02456(11) \\ \mathrm{B} 124 & 0.35282(4) & 0.7500 & 0.93610(4) & 0.02254(13) \\ \mathrm{C} 131 & 0.3904(3) & 0.2500 & 0.1747(3) & 0.0161(10) \\ \mathrm{C} 132 & 0.3885(2) & 0.3685(3) & 0.1192(3) & 0.0169(7) \\ \mathrm{C} 133 & 0.3821(2) & 0.3691(3) & 0.0100(2) & 0.0179(7) \\ \mathrm{H} 133 & 0.3806 & 0.4499 & -0.0281 & 0.021^{*} \\ \mathrm{C} 134 & 0.3781(3) & 0.2500 & -0.0428(4) & 0.0190(10) \\ \mathrm{N} 137 & 0.3955(3) & 0.2500 & 0.2840(3) & 0.0180(8) \\ \mathrm{C} 137 & 0.3995(3) & 0.2500 & 0.3761(4) & 0.0246(11) \\ \mathrm{B} 132 & 0.39399(3) & 0.52885(3) & 0.19404(3) & 0.02480(11) \\ \mathrm{B} 134 & 0.36801(4) & 0.2500 & -0.19267(4) & 0.02564(14) \\ & & & & \end{array}$

Atomic displacement parameters $\left(\AA^{2}\right)$

\begin{tabular}{lllllll}
\hline & $U^{11}$ & $U^{22}$ & $U^{33}$ & $U^{12}$ & $U^{13}$ & $U^{23}$ \\
\hline $\mathrm{C} 121$ & $0.013(2)$ & $0.019(2)$ & $0.016(2)$ & 0.000 & $0.0002(19)$ & 0.000 \\
$\mathrm{C} 122$ & $0.0164(16)$ & $0.0157(16)$ & $0.0207(17)$ & $0.0016(14)$ & $-0.0016(14)$ & $-0.0031(13)$ \\
$\mathrm{C} 123$ & $0.0191(17)$ & $0.0152(17)$ & $0.0202(17)$ & $0.0013(14)$ & $-0.0017(14)$ & $0.0039(13)$ \\
$\mathrm{C} 124$ & $0.017(2)$ & $0.017(2)$ & $0.018(2)$ & 0.000 & $0.0003(19)$ & 0.000 \\
$\mathrm{~N} 127$ & $0.026(2)$ & $0.018(2)$ & $0.021(2)$ & 0.000 & $0.0008(17)$ & 0.000 \\
$\mathrm{C} 127$ & $0.035(3)$ & $0.025(3)$ & $0.026(3)$ & 0.000 & $0.002(2)$ & 0.000 \\
Br122 & $0.0339(2)$ & $0.01579(18)$ & $0.0239(2)$ & $-0.00052(15)$ & $0.00210(15)$ & $-0.00522(14)$ \\
Br124 & $0.0290(3)$ & $0.0231(3)$ & $0.0155(2)$ & 0.000 & $-0.0005(2)$ & 0.000 \\
C131 & $0.014(2)$ & $0.019(2)$ & $0.015(2)$ & 0.000 & $-0.0026(17)$ & 0.000 \\
C132 & $0.0158(16)$ & $0.0138(16)$ & $0.0210(17)$ & $0.0001(13)$ & $0.0003(13)$ & $-0.0042(13)$ \\
C133 & $0.0229(18)$ & $0.0132(17)$ & $0.0174(17)$ & $0.0014(14)$ & $-0.0001(13)$ & $0.0035(13)$ \\
C134 & $0.018(2)$ & $0.024(3)$ & $0.015(2)$ & 0.000 & $-0.0016(18)$ & 0.000 \\
N137 & $0.019(2)$ & $0.017(2)$ & $0.018(2)$ & 0.000 & $0.0008(16)$ & 0.000 \\
C137 & $0.024(3)$ & $0.019(3)$ & $0.030(3)$ & 0.000 & $-0.001(2)$ & 0.000 \\
Br132 & $0.0360(2)$ & $0.01500(19)$ & $0.0234(2)$ & $-0.00111(15)$ & $-0.00260(14)$ & $-0.00427(14)$ \\
Br134 & $0.0393(3)$ & $0.0222(3)$ & $0.0154(3)$ & 0.000 & $0.0003(2)$ & 0.000 \\
& & & & & & \\
\hline
\end{tabular}

Geometric parameters $\left(\AA,{ }^{\circ}\right)$

\begin{tabular}{llll}
\hline $\mathrm{C} 121-\mathrm{N} 127$ & $1.393(6)$ & $\mathrm{C} 131-\mathrm{N} 137$ & $1.380(6)$ \\
$\mathrm{C} 121-\mathrm{C} 122^{\mathrm{i}}$ & $1.395(4)$ & $\mathrm{C} 131-\mathrm{C} 132^{\mathrm{ii}}$ & $1.392(4)$ \\
$\mathrm{C} 122-\mathrm{C} 123$ & $1.382(4)$ & $\mathrm{C} 132-\mathrm{C} 133$ & $1.380(4)$ \\
$\mathrm{C} 122-\mathrm{B} 122$ & $1.882(3)$ & $\mathrm{C} 132-\mathrm{B} 132$ & $1.883(3)$ \\
$\mathrm{C} 123-\mathrm{C} 124^{\mathrm{i}}$ & $1.381(4)$ & $\mathrm{C} 133-\mathrm{C} 134^{\mathrm{ii}}$ & $1.381(4)$ \\
$\mathrm{C} 123-\mathrm{H} 123$ & 0.9500 & $\mathrm{C} 133-\mathrm{H} 133$ & 0.9500 \\
$\mathrm{C} 124-\mathrm{Br} 124$ & $1.897(5)$ & $\mathrm{C} 134-\mathrm{Br} 134$ & $1.895(4)$ \\
$\mathrm{N} 127-\mathrm{C} 127$ & $1.147(6)$ & $\mathrm{N} 137-\mathrm{C} 137$ & $1.164(6)$ \\
$\mathrm{N} 127-\mathrm{C} 121-\mathrm{C} 122^{\mathrm{i}}$ & & & \\
$\mathrm{C} 122-\mathrm{C} 121-\mathrm{C} 122^{\mathrm{i}}$ & $120.4(2)$ & $\mathrm{N} 137-\mathrm{C} 131-\mathrm{C} 132^{\mathrm{ii}}$ & $120.3(2)$ \\
& $119.1(4)$ & $\mathrm{C} 132^{\mathrm{ii}}-\mathrm{C} 131-\mathrm{C} 132$ & $119.5(4)$
\end{tabular}




$\begin{array}{llll}\mathrm{C} 123-\mathrm{C} 122-\mathrm{C} 121 & 120.8(3) & \mathrm{C} 133-\mathrm{C} 132-\mathrm{C} 131 & 120.5(3) \\ \mathrm{C} 123-\mathrm{C} 122-\mathrm{B} 122 & 119.6(2) & \mathrm{C} 133-\mathrm{C} 132-\mathrm{B} 132 & 119.9(2) \\ \mathrm{C} 121-\mathrm{C} 122-\mathrm{B} 122 & 119.6(2) & \mathrm{C} 131-\mathrm{C} 132-\mathrm{B} 132 & 119.5(2) \\ \mathrm{C} 124-\mathrm{C} 123-\mathrm{C} 122 & 118.3(3) & \mathrm{C} 132-\mathrm{C} 133-\mathrm{C} 134 & 118.7(3) \\ \mathrm{C} 124-\mathrm{C} 123-\mathrm{H} 123 & 120.8 & \mathrm{C} 132-\mathrm{C} 133-\mathrm{H} 133 & 120.7 \\ \mathrm{C} 122-\mathrm{C} 123-\mathrm{H} 123 & 120.8 & \mathrm{C} 134-\mathrm{C} 133-\mathrm{H} 133 & 120.7 \\ \mathrm{C} 123-\mathrm{C} 124-\mathrm{C} 123 & \mathrm{C} 1333^{\mathrm{i}}-\mathrm{C} 134-\mathrm{C} 133 & 122.1(4) \\ \mathrm{C} 123-\mathrm{C} 124-\mathrm{B} 124 & 122.5(4) & \mathrm{C} 133^{\mathrm{i}}-\mathrm{C} 134-\mathrm{Br} 134 & 118.9(2) \\ \mathrm{C} 127-\mathrm{N} 127-\mathrm{C} 121 & 118.7(2) & \mathrm{C} 137-\mathrm{N} 137-\mathrm{C} 131 & 179.8(4) \\ & 178.5(5) & & \\ \mathrm{N} 127-\mathrm{C} 121-\mathrm{C} 122-\mathrm{C} 123 & 176.7(3) & \mathrm{N} 137-\mathrm{C} 131-\mathrm{C} 132-\mathrm{C} 133 & -179.2(3) \\ \mathrm{C} 122-\mathrm{C} 121-\mathrm{C} 122-\mathrm{C} 123 & -1.0(6) & \mathrm{C} 132^{\mathrm{ii}}-\mathrm{C} 131-\mathrm{C} 132-\mathrm{C} 133 & 1.6(6) \\ \mathrm{N} 127-\mathrm{C} 121-\mathrm{C} 122-\mathrm{Br} 122 & -3.0(5) & \mathrm{N} 137-\mathrm{C} 131-\mathrm{C} 132-\mathrm{Br} 132 & 0.6(5) \\ \mathrm{C} 122-\mathrm{C} 121-\mathrm{C} 122-\mathrm{B} 122 & 179.30(19) & \mathrm{C} 132-\mathrm{C} 131-\mathrm{C} 132-\mathrm{Br} 132 & -178.6(2) \\ \mathrm{C} 121-\mathrm{C} 122-\mathrm{C} 123-\mathrm{C} 124 & -0.5(5) & \mathrm{C} 131-\mathrm{C} 132-\mathrm{C} 133-\mathrm{C} 134 & -0.2(5) \\ \mathrm{B} 122-\mathrm{C} 122-\mathrm{C} 123-\mathrm{C} 124 & 179.2(3) & \mathrm{Br} 132-\mathrm{C} 132-\mathrm{C} 133-\mathrm{C} 134 & -179.9(3) \\ \mathrm{C} 122-\mathrm{C} 123-\mathrm{C} 124-\mathrm{C} 123^{\mathrm{i}} & 2.1(7) & \mathrm{C} 132-\mathrm{C} 133-\mathrm{C} 134-\mathrm{C} 133^{\mathrm{ii}} & -1.3(7) \\ \mathrm{C} 122-\mathrm{C} 123-\mathrm{C} 124-\mathrm{Br} 124 & -177.4(2) & \mathrm{C} 132-\mathrm{C} 133-\mathrm{C} 134-\mathrm{Br} 134 & 179.3(3)\end{array}$

Symmetry codes: (i) $x,-y+3 / 2, z$; (ii) $x,-y+1 / 2, z$.

Hydrogen-bond geometry $\left(\AA,{ }^{\circ}\right)$

\begin{tabular}{lllll}
\hline$D-\mathrm{H} \cdots A$ & $D-\mathrm{H}$ & $\mathrm{H} \cdots A$ & $D \cdots A$ & $D-\mathrm{H} \cdots A$ \\
\hline $\mathrm{C} 123-\mathrm{H} 123 \cdots \mathrm{Br} 134^{\mathrm{iii}}$ & 0.95 & 3.08 & $3.976(3)$ & 157 \\
$\mathrm{C} 133-\mathrm{H} 133 \cdots \mathrm{Br} 124^{\mathrm{iv}}$ & 0.95 & 3.10 & $3.995(3)$ & 157 \\
\hline
\end{tabular}

Symmetry codes: (iii) $x, y, z+1$; (iv) $x, y, z-1$. 